\title{
Estrogen receptor- $\alpha$ in medial amygdala neurons regulates body weight
}

\author{
Pingwen Xu, ${ }^{1}$ Xuehong Cao, ${ }^{1}$ Yanlin He, ${ }^{1}$ Liangru Zhu ${ }^{1,2}$ Yongjie Yang, ${ }^{1}$ Kenji Saito, ${ }^{1}$ Chunmei Wang, ${ }^{1}$ Xiaofeng Yan, ${ }^{1}$ \\ Antentor Othrell Hinton Jr., ${ }^{1}$ Fang Zou, ${ }^{1}$ Hongfang Ding, ${ }^{1}$ Yan Xia, ${ }^{1}$ Chunling Yan, ${ }^{1}$ Gang Shu, ${ }^{1}$ San-Pin Wu, ${ }^{3}$ Bin Yang, ${ }^{4}$ \\ Yuxin Feng, ${ }^{5}$ Deborah J. Clegg, ${ }^{6}$ Richard DeMarchi, ${ }^{4}$ Sohaib A. Khan, ${ }^{7}$ Sophia Y. Tsai, ${ }^{3}$ Francesco J. DeMayo, ${ }^{3}$ Qi Wu, ${ }^{1}$ \\ Qingchun Tong, ${ }^{8}$ and Yong $\mathrm{Xu}^{1,3}$
}

'Children's Nutrition Research Center, Department of Pediatrics, Baylor College of Medicine, Houston, Texas, USA. ²Department of Gastroenterology, Union Hospital, Tongji Medical College, Huazhong University of Science and Technology, Wuhan, China. ${ }^{3}$ Department of Molecular and Cellular Biology, Baylor College of Medicine, Houston, Texas, USA. ${ }^{4}$ Department of Chemistry, Indiana University, Bloomington, Indiana, USA. ${ }^{5}$ Cincinnati Children's Hospital Medical Center, Cincinnati, Ohio, USA. ${ }^{6}$ Department of Biomedical Research, Diabetes and Obesity Research Institute, Cedars-Sinai Medical Center, Los Angeles, California, USA. 'Department of Cell and Cancer Biology, Vontz Center for Molecular Studies, University of Cincinnati College of Medicine, Cincinnati, Ohio, USA. ${ }^{8}$ Brown Foundation Institute of Molecular Medicine, University of Texas Health Science Center at Houston, Houston, Texas, USA.

\begin{abstract}
Estrogen receptor- $\alpha(E R \alpha)$ activity in the brain prevents obesity in both males and females. However, the ER $\alpha$-expressing neural populations that regulate body weight remain to be fully elucidated. Here we showed that single-minded-1 (SIM1) neurons in the medial amygdala (MeA) express abundant levels of ER $\alpha$. Specific deletion of the gene encoding ER $\alpha$ (Esr1) from SIM1 neurons, which are mostly within the MeA, caused hypoactivity and obesity in both male and female mice fed with regular chow, increased susceptibility to diet-induced obesity (DIO) in males but not in females, and blunted the body weightlowering effects of a glucagon-like peptide-1-estrogen (GLP-1-estrogen) conjugate. Furthermore, selective adeno-associated virus-mediated deletion of Esr1 in the MeA of adult male mice produced a rapid body weight gain that was associated with remarkable reductions in physical activity but did not alter food intake. Conversely, overexpression of ER $\alpha$ in the MeA markedly reduced the severity of DIO in male mice. Finally, an ER $\alpha$ agonist depolarized MeA SIM1 neurons and increased their firing rate, and designer receptors exclusively activated by designer drug-mediated (DREADD-mediated) activation of these neurons increased physical activity in mice. Collectively, our results support a model where ER $\alpha$ signals activate MeA neurons to stimulate physical activity, which in turn prevents body weight gain.
\end{abstract}

\section{Introduction}

Estrogens play critical roles in the regulation of energy homeostasis. Depletion of endogenous estrogens in female rodents, via ovariectomy (OVX), causes increased body weight and hyperadiposity (1, 2). 17ß-estradiol replacement can prevent obesity in OVX female animals (1). The body weight-lowering effects of estrogens are attributed both to decreased food intake and increased energy expenditure (3). These estrogenic actions are primarily mediated by estrogen receptor- $\alpha$ (ER $\alpha$, encoded by ESRl) because the deletion of Esr1, but not estrogen receptor $\beta$ (Esr 2 ), leads to increased body weight and adiposity in both male and female mice (4-6). Recently, we have demonstrated that deletion of Esr 1 from the entire brain results in obesity due to both hyperphagia and decreased energy expenditure (7), which indicates that the estrogenic effects on energy homeostasis require activation of brain $\mathrm{ER} \alpha$. We and others further genetically isolated the distinct functions of 2 critical ER $\alpha$-expressing neural populations: pro-opiomelanocortin (POMC) neurons in the arcuate nucleus of

\section{Related Commentary: p. 2576}

Authorship note: Pingwen Xu and Xuehong Cao contributed equally to this work. Conflict of interest: The authors have declared that no conflict of interest exists. Submitted: January 12, 2015; Accepted: May 8, 2015.

Reference information: J Clin Invest. 2015;125(7):2861-2876. doi:10.1172/JCI80941. the hypothalamus (ARH) and steroidogenic factor-1 (SF1) neurons in the ventromedial hypothalamic nucleus (VMH). Thus, loss of ER $\alpha$ expressed by ARH POMC neurons in females causes hyperphagia but does not directly affect energy expenditure (7). On the other hand, deletion of Esr1 in female VMH neurons leads to decreased energy expenditure associated with reductions in the resting metabolic rate and thermogenesis, but this deletion does not affect food intake (7-9). In addition, ER $\alpha$ expressed in the nucleus of solitary tract (NTS) of the brainstem (10), in the preoptic anterior hypothalamus (POAH) (11), and in the dorsal raphe nucleus (DRN) $(11,12)$ is implicated in the regulation of feeding in females.

Although we and others have substantially narrowed down the relevant $\mathrm{ER} \alpha$ sites mediating metabolic effects of estrogens, the functional mapping of $\mathrm{ER} \alpha$ in the brain is incomplete. For instance, the brain ER $\alpha$ is clearly required for the regulation of body weight in male mice (7), but the exact ER $\alpha$ population that controls male energy homeostasis remains unknown. Additionally, while deletion of Esr 1 from the entire brain leads to decreased physical activity, mice lacking $\mathrm{ER} \alpha$ selectively in POMC and/or SF1 neurons showed normal physical activity (7). Together, these findings suggest that other brain ER $\alpha$ sites must exist to regulate male energy homeostasis and/or regulate physical activity.

$\mathrm{ER} \alpha$ is abundantly expressed in the medial amygdala (MeA) (13), a brain structure outside the hypothalamus. Earlier studies 
showed that injections of $17 \beta$-estradiol into the amygdala decrease body weight in rats, which is an effect that remains in rats with large hypothalamic lesions (14). These findings suggest that ER $\alpha$ in the MeA may regulate energy balance. Here we generated mouse models with ER $\alpha$ selectively deleted or overexpressed in the MeA. Using these tools, we examined the physiological functions of MeA ER $\alpha$ in the context of energy homeostasis in both male and female mice. We also recorded effects of an ER $\alpha$ agonist on the electrophysiological properties of MeA neurons. Finally, we used the designer receptors exclusively activated by designer drugs (DREADD) approach $(15,16)$ to examine effects of MeA neural activity on energy balance in mice.

\section{Results}

ERa colocalizes with SIM1 primarily in MeA neurons. Singleminded-1 (SIM1) is a helix-loop-helix transcriptional factor, which plays an important role in body weight control (17-19). A Sim1-Cre transgenic mouse line expresses abundant Cre recombinase in multiple brain regions, including the MeA (20). Using Sim1-Cre Rosa26-GFP mice in which all SIM1-expressing neurons are labeled with GFP, we systemically examined colocalization of ER $\alpha$ and SIM1 (GFP) in both male and female brains. In the MeA, we found that about $40 \%$ of ER $\alpha$ neurons $(38.4 \pm 1.2 \%$ in females and $43.7 \pm 1.9 \%$ in males) coexpress SIM1 (Figure 1, A, B, I, and J). An interesting sexual dimorphism was also observed with a trend of more ER $\alpha$-expressing SIM1 neurons in male MeA than in female MeA (Figure 1, I and J; male: 6,750 \pm 951 vs. female: 4,176 \pm 398 ; $P=0.06)$. While abundant SIM1 and ER $\alpha$ are also found in the $\mathrm{POAH}$, these 2 populations are largely segregated in this region, with only few neurons coexpressing SIM1 and ER $\alpha$ (Figure 1, C, I, and J). The paraventricular hypothalamic nucleus (PVN) expresses abundant SIM1 but low levels of ER $\alpha$, with scattered neurons coexpressing SIM1 and ER $\alpha$ (Figure 1, D, I, and J). SIM1 neurons are also found in other brain regions, such as posterior hypothalamic area $(\mathrm{PH})$, nucleus of the lateral olfactory tract (LOT), supraoptic nucleus (SON), and periaqueductal gray (PAG), but none of these SIM1 neurons coexpress ER $\alpha$ (Figure 1, E-H). In summary, we found that SIM1 colocalizes with ER $\alpha$ only in 3 brain regions, the MeA, POAH, and PVN. In particular, most of these SIM1/ER $\alpha$ double-labeled neurons are located within the $\mathrm{MeA}(80 \pm 2.4 \%$ in males and $79.2 \pm 1.7 \%$ in females) (Figure $1 \mathrm{~K}$ ).

Loss of ER in SIM1 neurons leads to hypoactivity and obesity in females. We crossed Esr $1^{f / f l}$ mice (21) and Sim1-Cre mice (20) to generate mice lacking ER $\alpha$ only in SIM1 neurons (Sim1-Cre Esr $1^{f / f}$, herein referred to as SIM1-ER $\alpha-\mathrm{KO}$ ). Based on the distribution pattern of SIM1/ER $\alpha$ double-labeled neurons in WT brains (Figure $1 \mathrm{~K})$, we predict that SIM1-ER $\alpha-\mathrm{KO}$ mice have Esr1 deleted primarily in the MeA, with modest deletion in the POAH and PVN. In order to validate selective deletion of Esr1 from SIM1 neurons, we compared ER $\alpha$ expression in Sim1-Cre Esr $1^{f / f l}$ Rosa26-GFP (herein referred to as SIM1-ER $\alpha-\mathrm{KO}-\mathrm{GFP}$ ) and Sim1-Cre Rosa26-GFP (control) mice. Notably, while abundant ER $\alpha$ is expressed by MeA SIM1 neurons in control mice (Supplemental Figure 1, A, C, and D; supplemental material available online with this article; doi:10.1172/ JCI80941DS1), nearly no MeA SIM1 neurons in SIM1-ER $\alpha-K O-G F P$ mice coexpress ER $\alpha$ (Supplemental Figure 1, B-D). Importantly, abundant ER $\alpha$ was observed in non-SIM1 MeA neurons in SIM1-
ER $\alpha$-KO-GFP mice (Supplemental Figure 1B), indicating that deletion of Esr 1 is restricted in SIM1 population. In addition, the numbers of MeA SIM1 neurons (GFP neurons) in control and SIM1-ER $\alpha-\mathrm{KO}-$ GFP mice are comparable (Supplemental Figure 1, A-D), indicating that loss of ER $\alpha$ does not cause unwanted cell death.

We then systemically characterized the metabolic phenotypes of female SIM1-ER $\alpha$-KO mice, and their control littermates fed on regular chow. We found that female SIM1-ER $\alpha$-KO mice developed late-onset obesity when fed chow. Notably, female SIM1$\mathrm{ER} \alpha-\mathrm{KO}$ mice started to be significantly heavier than their controls at the age of 22 weeks, and by 26 weeks of age, SIM1-ER $\alpha-K O$ females gained $5.0 \mathrm{~g}$ more body weight than controls (about 20\% of control body weight) (Figure 2A). This weight difference was solely due to increases in fat mass, while lean mass of 2 groups was comparable (Figure 2B). Consistently, 2-fold increases in both gonadal white adipose tissue (gWAT) and inguinal WAT (iWAT) were observed in SIM1-ER $\alpha$-KO females compared with control littermates (Figure 2C). Furthermore, the average adipocyte sizes of both gWAT and iWAT were significantly larger in SIM1-ER $\alpha$-KO females than in control females (Figure 2, D and E). Consistent with increased adiposity, we observed a significant increase in serum leptin levels in SIM1-ER $\alpha$-KO females (Supplemental Table 1). These data indicate that ER $\alpha$ expressed by SIM1 neurons in female brains is required to maintain normal body weight and adiposity at a later age.

To determine the mechanisms underlying the obese phenotypes seen in SIM1-ER $\alpha$-KO female mice, another cohort of chowfed SIM1-ER $\alpha$-KO females and control littermates ( 5 months of age), with matched body weight and lean mass, were adapted into the Comprehensive Lab Animal Monitoring System (CLAMS) metabolic chambers to measure food intake and energy expenditure. Female SIM1-ER $\alpha$-KO mice consumed comparable food as control mice (Figure 3A). In contrast, a modest but significant decrease in energy expenditure was observed, primarily during the dark cycle, in female SIM1-ER $\alpha$-KO mice (Figure 3B).

Since energy expenditure is contributed by 3 components physical activities, resting metabolic rate, and thermogenesis (22) - we further dissected these 3 components in female SIM1-ER $\alpha-K O$ mice. Remarkably, physical activities (demonstrated by ambulatory movements and rearing activities) were robustly reduced, primarily during the dark cycle, in female SIM1-ER $\alpha-\mathrm{KO}$ mice compared with their controls (Figure 3, C-F). On the other hand, the resting metabolic rate, as assessed by measuring the minimal energy expenditure during the light cycle (23), was comparable between female SIM1-ER $\alpha$-KO and control mice (Figure 3G). Notably, estrogens have been shown to stimulate energy expenditure partly through enhancing thermogenesis in the brown adipose tissue (BAT) (9). However, female SIM1-ER $\alpha$-KO mice expressed comparable levels of thermogenic genes in the BAT, including $\beta 3$ adrenergic receptor (Adrb3), Cebpa, Esrra, Pgc1a, Pparg, Prdm16, Rip140, and Ucp3 (Figure 3H). Interestingly, a significant increase in $U c p 1$ expression was observed in the BAT of SIM1-ER $\alpha$-KO compared with control females (Figure $3 \mathrm{H}$ ), which may have been a compensatory response in these mutant mice to prevent further body weight gain. Taken together, our results indicate that loss of ER $\alpha$ in SIM1 neurons led to increased body weight gain in female mice, associated with profound decreases in physical activity and modest reductions in energy expenditure. 

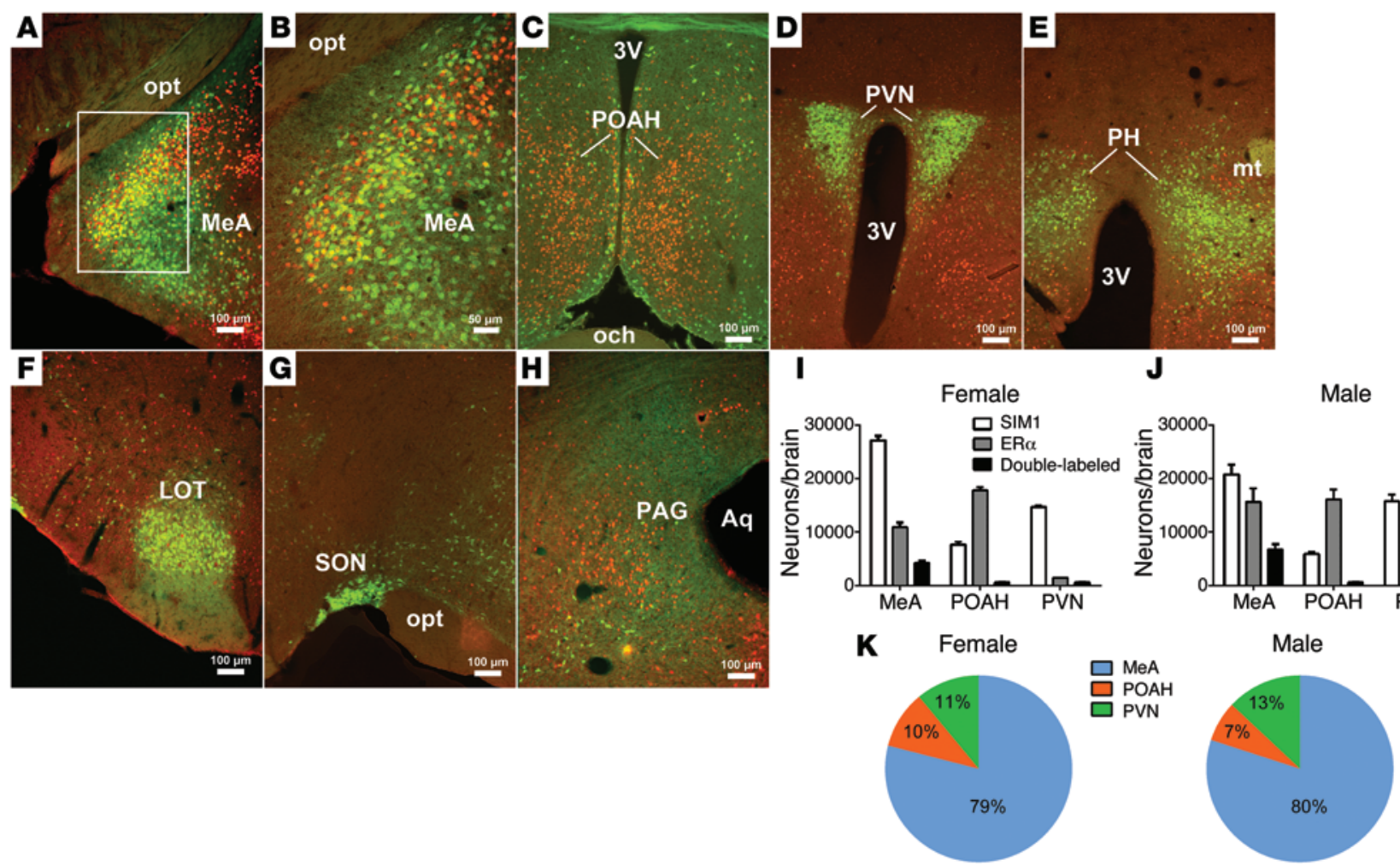

Figure 1. Colocalization of ER $\alpha$ and SIM1 in mouse brains. (A-H) Dual immunofluorescence for GFP (green) and ER $\alpha$ (red) in the MeA (A and B), POAH (C), PVN (D), PH (E), LOT (F), SON (G), and PAC (H) in Sim1-Cre Rosa26-GFP mouse brains. B is higher magnification of the white box in A. 3V, 3rd ventricle; Aq, aqueduct; $\mathrm{mt}$, mammillothalamic tract; och, optic chiasm; opt, optic tract. (I and J) Numbers of neurons expressing SIM1 (GFP), ER $\alpha$, and both in the MeA, $\mathrm{POAH}$, and PVN in female (I) and male (J) mice. Data are presented as mean \pm SEM. $n=3 /$ group. (K) Percentage distribution of ER $\alpha / \mathrm{SIM} 1$ double-labeled neurons in the MeA, POAH, and PVN. Data are presented as mean \pm SEM. $n=3 /$ group.

Loss of ERa in SIM1 neurons leads to hypoactivity and obesity in males. Similar to female SIM1-ER $\alpha$-KO mice, male mutants also showed late-onset obesity when fed chow. In particular, male SIM1-ER $\alpha$-KO mice started to be significantly heavier than their controls at the age of 26 weeks, and by 32 weeks of age, SIM1-ER $\alpha$-KO males gained $5.3 \mathrm{~g}$ more body weight than controls (about $15 \%$ of control body weight) (Figure 4A). A significant increase in fat mass, but not lean mass, was observed (Figure 4B). Consistently, the weight and cell size of gWAT and iWAT were significantly increased in SIM1-ER $\alpha$-KO males compared with controls (Figure 4, C-E).

Interestingly, while food intake of SIM1-ER $\alpha$-KO males was not altered (Figure 5A), a modest but significant reduction in energy expenditure was observed at multiple time points during the 24-hour recording (Figure 5B), associated with reductions in ambulation and rearing (Figure 5, C-F). No significant changes were observed in resting metabolic rate (Figure 5G) and BAT thermogenic gene expression, including Ucp1 (Figure 5H). Collectively, these data indicate that, similar to females, loss of ER $\alpha$ in SIM1 neurons also led to increased body weight gain in male mice at a later age, associated with decreased physical activity and reductions in energy expenditure.

Other phenotypes in SIM1-ER $\alpha-K O$ mice. We analyzed gene expression profile in the amygdala, and no significant changes were observed in mRNA levels of androgen receptor $(A r)$, aromatase, glutamate decarboxylase 1 ( $G a d 1$ ), glutamate decarboxylase
2 (Gad2), glutaminase $(G l s)$, glutamate-ammonia ligase (Glul), melanocortin 4 receptor $(M c 4 r)$, and nitric oxide synthase-1 (Nos1) in either male or female SIM1-ER $\alpha$-KO mice compared with their controls (Supplemental Figure 2, A and B). Similarly, no changes were detected in agouti-related peptide (Agrp), Esr1, leptin receptor, Nos1, neuropeptide Y (Npy), and Pomc mRNAs in the hypothalamus (Supplemental Figure 2, C and D).

Since the amygdala has been heavily implicated in the regulation of anxiety, we characterized the anxiety levels in both male and female SIM1-ER $\alpha$-KO mice in various behavioral tests during the light cycle. In the open field test, a significant decrease in distance traveled was observed during the early phase of the test in SIM1-ER $\alpha$-KO males but not in females, and this difference dissipated in the late phase of the test (Supplemental Figure 3, A and $\mathrm{B}$ ). No difference was detected in center time and the numbers of fecal boli (Supplemental Figure 3, C and D). No changes were detected in various parameters either in the light and dark field test (Supplemental Figure 4) or in the the elevated plus maze test (Supplemental Figure 5). While these results indicate that ER $\alpha$ expressed by SIM1 neurons is not essential for the regulation of anxiety, we cannot rule out potential roles of ER $\alpha$ expressed by non-SIM1 neurons in the MeA in the context of anxiety.

We characterized endocrine functions of male or female SIM1-ER $\alpha$-KO mice and found no alterations in serum triiodothyronine, thyroxine, norepinephrine, epinephrine, testosterone, and 17ß-estradiol levels (Supplemental Table 1). Furthermore, 

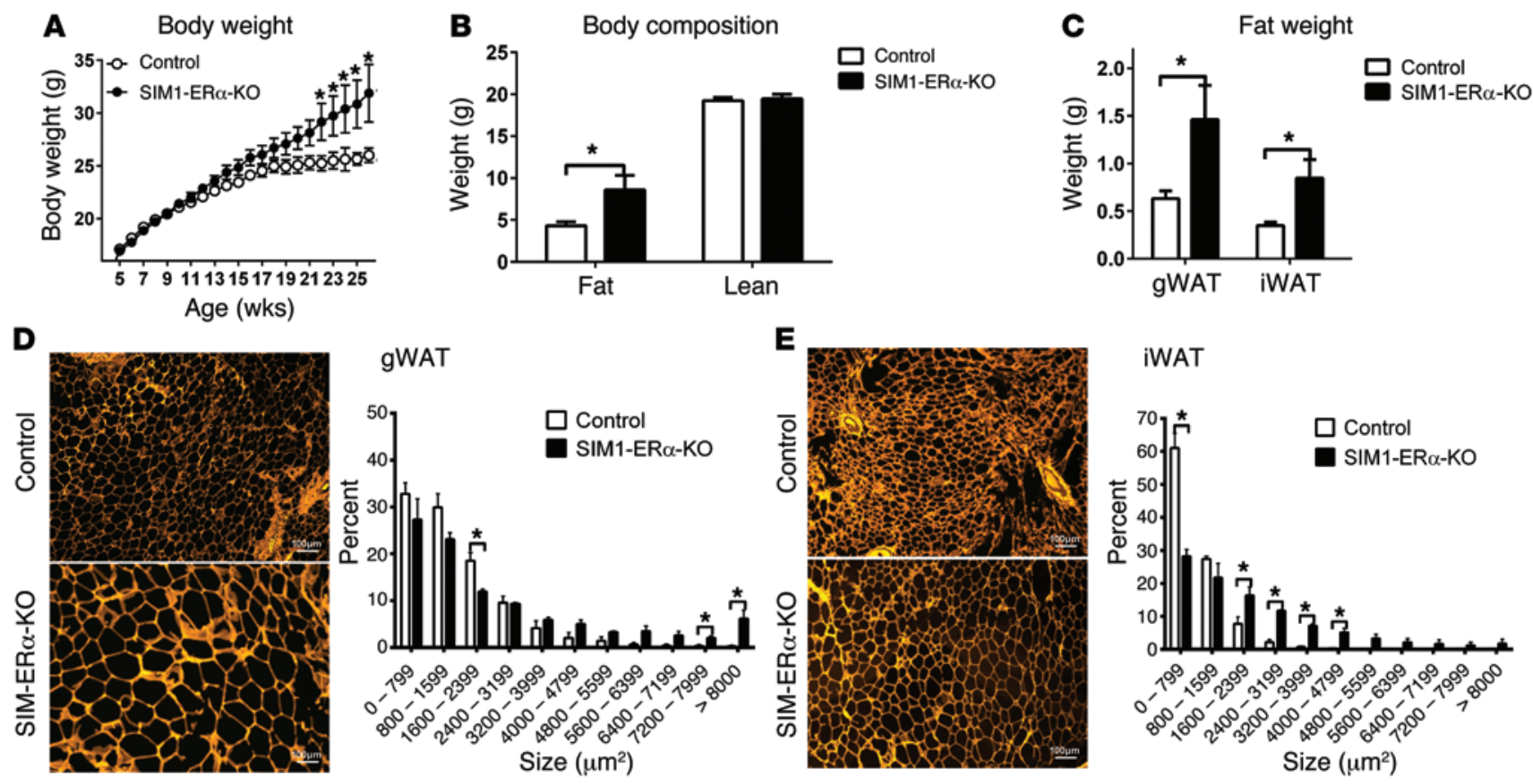

Figure 2. Obesity in chow-fed female SIM1-ER $\alpha-K O$ mice. (A) Body weight of female control and SIM1-ER $\alpha$-KO littermates weaned on chow. Data are presented as mean \pm SEM. $n=27$ or 30/group. ${ }^{*} P<0.05$ in 2-way ANOVA analysis followed by post hoc Bonferroni tests. (B) Body fat mass and lean mass measured at 26 weeks of age. Data are presented as mean \pm SEM. $n=27$ or 30/group. ${ }^{*} P<0.05$ in $t$ test. (C) Weights of gWAT and iWAT at 26 weeks of age. Data are presented as mean \pm SEM. $n=8$ /group. ${ }^{*} P<0.05$ in $t$ test. (D and E) Cell size in gWAT(D) and iWAT (E) pads at 26 weeks of age. Data are presented as mean \pm SEM. $n=3$ /group. ${ }^{*} P<0.05$ in $t$ test.

SIM1-ER $\alpha$-KO female mice displayed normal onset of vaginal opening and estrus (Supplemental Figure 6, A and B), and normal estrus cyclicity (Supplemental Figure 6C). There was no difference in breeding success rate, litter size, or birth latency between control and SIM1-ER $\alpha$-KO dams (Supplemental Figure 6, D-F). No deficits in fertility were observed in SIM1-ER $\alpha-\mathrm{KO}$ sires (data not shown).

Loss of ERa in SIM1 neurons increases susceptibility to dietinduced obesity in males but not in females. We then characterized body weight phenotypes in male and female SIM1-ER $\alpha-K O$ mice that were weaned on a high-fat diet (HFD). To our surprise, when fed on HFD up to 17 weeks of age, female SIM1-ER $\alpha$-KO showed comparable body weight, fat/lean mass, and food intake as control females (Figure 6,A-C). Energy expenditure was estimated by evaluating feeding efficiency (the ratio between body weight gain and cumulative food intake), and no changes were observed in female mice (Figure 6D). On the other hand, HFD-fed SIM1-ER $\alpha-\mathrm{KO}$ males showed significant increases in body weight compared with control males (Figure 6E). In particular, HFD-fed SIM1-ER $\alpha-\mathrm{KO}$ males started to be significantly heavier than their controls at the age of 14 weeks, and by 17 weeks of age, SIM1-ER $\alpha$-KO males gained $5.8 \mathrm{~g}$ more body weight than controls (about $15 \%$ of control body weight) (Figure 6E). Fat mass of SIM1-ER $\alpha-\mathrm{KO}$ males was significantly increased by 1-fold compared with control males, while lean mass was comparable between the 2 groups (Figure 6F). Cumulative food intake of male SIM1-ER $\alpha$-KO mice was comparable to that of control mice during most of the HFD feeding period and only became significantly higher after 16 weeks of HFD feeding (Figure 6G). Since hyperphagia developed only when body weight had already significantly diverged, we speculated that the increased food intake was the consequence, but not the cause, of obesity in these SIM1-ER $\alpha$-KO mice. On the other hand, feeding efficiency was significantly elevated in SIM1-ER $\alpha$-KO males, especially during the early phase of HFD feeding (Figure $6 \mathrm{H}$ ). These data indicate that loss of ER $\alpha$ in SIM1 neurons increases susceptibility to diet-induced obesity (DIO) in male mice, presumably by decreasing energy expenditure.

ER $\alpha$ in SIM1 neurons partly mediate body weight-lowering effects of GLP-1-estrogen conjugate. A GLP-1-estrogen conjugate was recently developed to preferentially deliver estrogens to GLP-1 receptorenriched regions (24). GLP-1-estrogen has been shown to substantially reduce body weight in both male and female mice with DIO, while the common side effects associated with estrogen therapy (e.g., reproductive endocrine toxicity and oncogenicity) are avoided (24). Interestingly, the MeA expresses considerable GLP-1 receptors (25). Furthermore, we showed that s.c. injections of GLP-1estrogen $(40 \mu \mathrm{g} / \mathrm{kg})$ significantly increased expression of tripartite motif-containing protein 25 (Trim25, a known estrogen target) in the amygdala of male mice, while a similar trend was also observed in the hypothalamus (Figure 6I). These results indicate that GLP1-estrogen conjugate delivers bioactive estrogens to the amygdala, in addition to other sites (e.g., the hypothalamus), and therefore raise the possibility that the body weight-lowering effects of GLP1-estrogen are partly mediated by ER $\alpha$ expressed by MeA neurons. To test this, we examined effects of GLP-1-estrogen and GLP-1 in male control and SIM1-ER $\alpha-\mathrm{KO}$ mice with DIO. Consistent with the original report (24), compared with GLP-1 (40 $\mu \mathrm{g} / \mathrm{kg} / \mathrm{d}$, s.c.) treatment, GLP-1-estrogen $(40 \mu \mathrm{g} / \mathrm{kg} / \mathrm{d}$, s.c.) produced a signifi- 
A

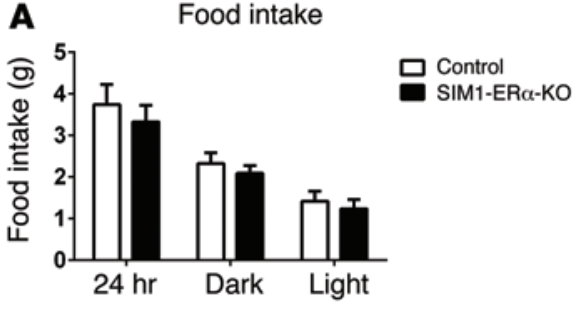

B

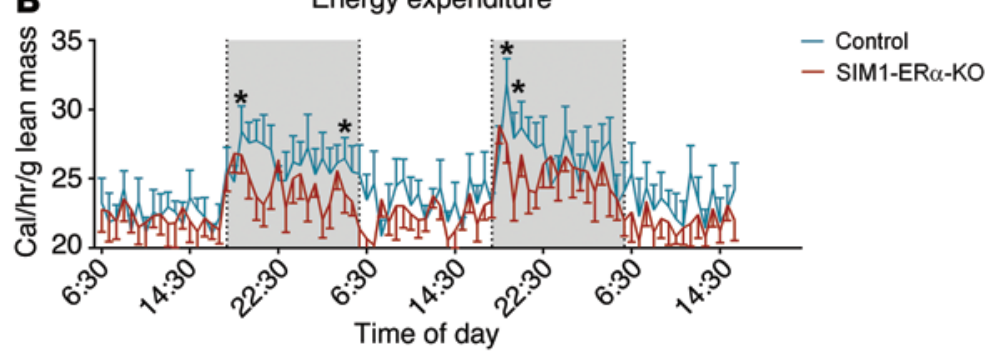

C

Ambulatory movement

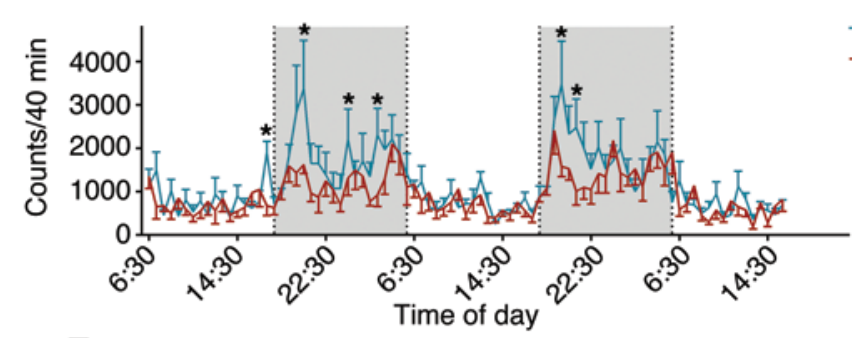

E

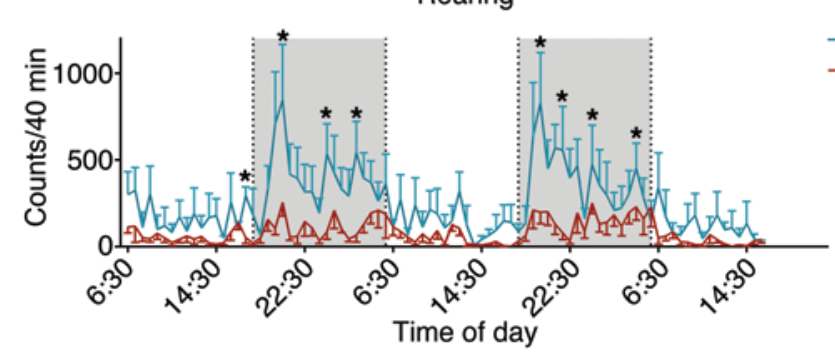

G

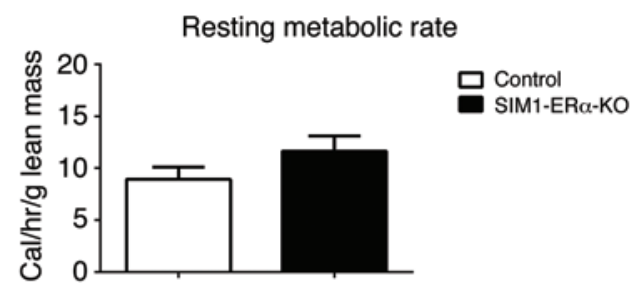

D Ambulatory movement
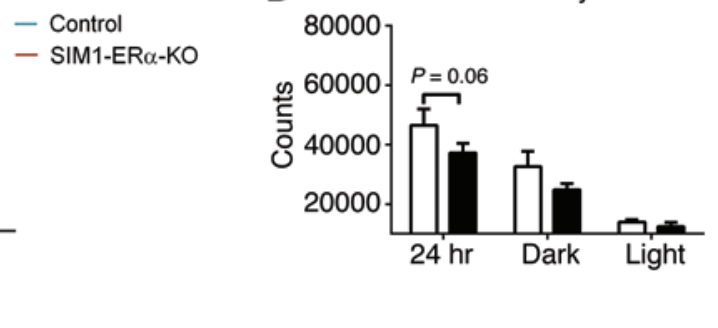

F Rearing

- Control
- SIM1-ER $\alpha-K O$

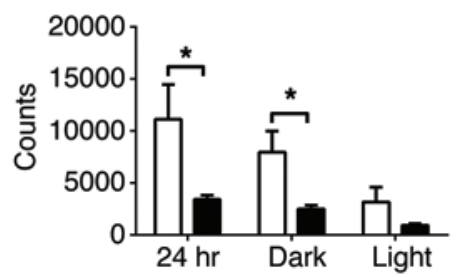

믄ol

SIM1-ER $\alpha$-KO

H

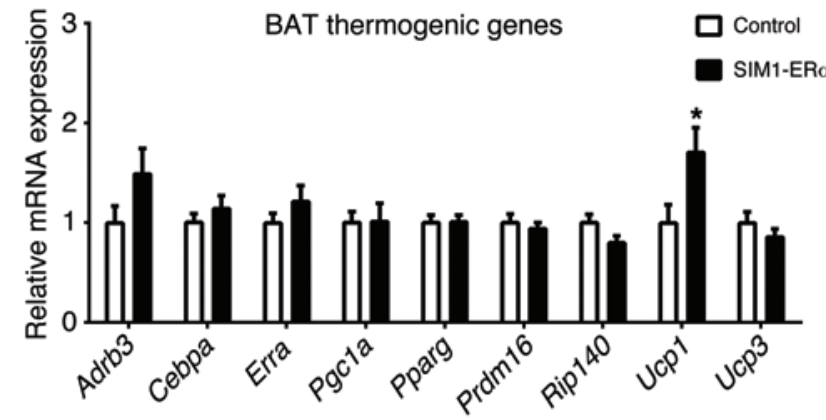

Figure 3. Metabolic profile of chow-fed female SIM1-ER $\boldsymbol{\alpha}$-KO mice. (A) Food intake during 24 hour, dark, or light cycles. (B) Energy expenditure. (C and D) Ambulatory movement. (E and F) Rearing activity. (C) Resting metabolic rate. Note that mice had comparable body weight (control: $26.4 \pm 1.1$ vs. SIM1-ER $\alpha-K O: 28.3 \pm 2.1, P>0.05$ ) and lean mass (control: $18.5 \pm 0.4$ vs. SIM1-ER $\alpha-K 0: 18.1 \pm 0.7, P>0.05$ ) at the time of the CLAMS study. (H) mRNA levels of thermogenic genes in BAT at 26 weeks of age. Data are presented as mean \pm SEM. $n=8 /$ group. ${ }^{*} P<0.05$ in $t$ test.

cantly more robust decrease in body weight in control mice (Figure 6J). In contrast, GLP-1-estrogen failed to significantly reduce body weight in SIM1-ER $\alpha$-KO mice compared with GLP-1-treated SIM1ER $\alpha$-KO mice (Figure 6J). Importantly, we found that body weight loss induced by GLP-1-estrogen tended to be attenuated in SIM1$\mathrm{ER} \alpha-\mathrm{KO}$ mice compared with control mice (Figure 6J), indicating that ER $\alpha$ in SIM1 neurons is at least partly required to mediate body weight-lowering effects of GLP-1-estrogen.

Deletion of Esr1 selectively in the MeA during adulthood led to rapid body weight gain in male mice. It is worth noting that in SIM1$\mathrm{ER} \alpha-\mathrm{KO}$ mice, Esr1 was deleted primarily in the MeA (80\%), with modest Esr1 deletions in the POAH (10\%) and PVN (10\%). Therefore, obesity observed in SIM1-ER $\alpha$-KO mice may result not only from loss of $\mathrm{ER} \alpha$ in the MeA, but also to some degree from loss of $\mathrm{ER} \alpha$ in the POAH/PVN. To further confirm the physiological functions of ER $\alpha$ in MeA neurons, we stereotaxically injected adeno-associated virus-Cre-GFP (AAV-Cre-GFP) into the MeA (bilaterally) of 12-week-old male Esrit/fll mice, which selectively deleted Esr1 in the MeA during adulthood (MeA-ER $\alpha$-KO mice). WT littermates received the same AAV-Cre-GFP stereotaxic injections and served as controls. Post hoc visualization of GFP was used to confirm accurate targeting to the MeA in each mouse (Figure 7A). Compared to control mice, $\mathrm{MeA}-\mathrm{ER} \alpha-\mathrm{KO}$ mice tended to gain more weight when fed chow (the first 8 days after virus injections), and this body weight gain became significantly different quickly after mice were switched to HFD (from day 8 until day 22), and MeA-ER $\alpha$-KO mice gained $4.9 \mathrm{~g}$ more body weight than controls on day 22 (Figure 7B). No differences in food intake were observed dur- 


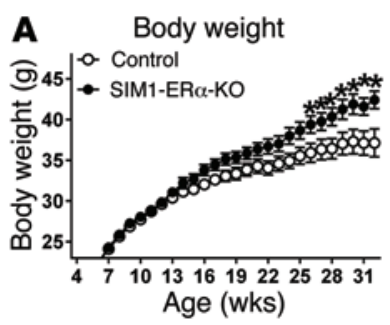

D

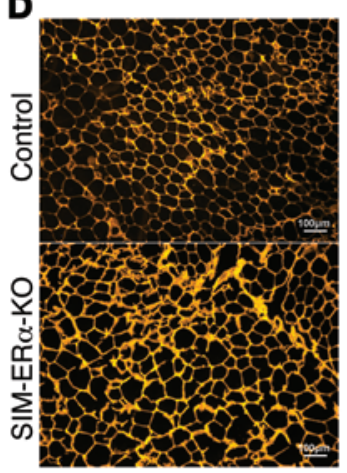

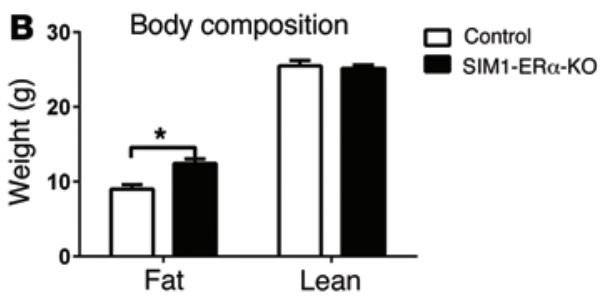

E

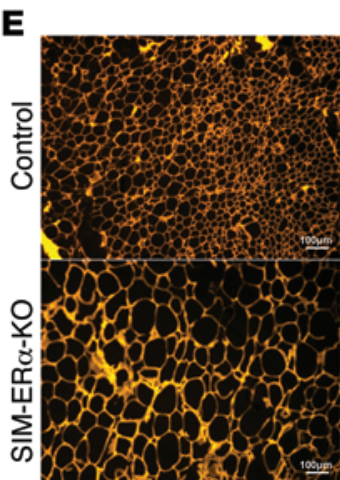

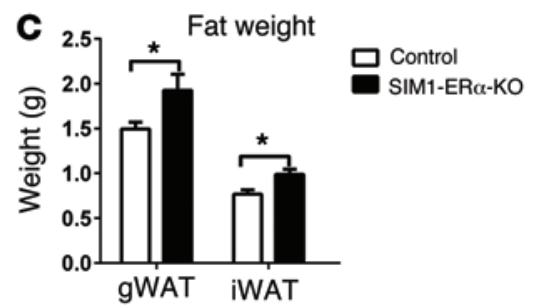

IWAT

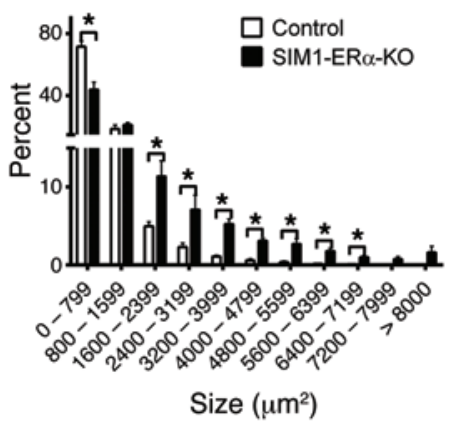

Figure 4. Obesity in chow-fed male SIM1-ER $\alpha$-KO mice. (A) Body weight of male control and SIM1-ER $\alpha$-KO littermates weaned on chow. Data are presented as mean \pm SEM. $n=26$ or 37/group. ${ }^{*} P<0.05$ in 2-way ANOVA analysis followed by post hoc Bonferroni tests. (B) Body fat mass and lean mass measured at 32 weeks of age. Data are presented as mean \pm SEM. $n=26$ or 37/group. ${ }^{*} P<0.05$ in $t$ test. (C) Weights of gWAT and iWAT at 32 weeks of age. Data are presented as mean \pm SEM. $n=8$ /group. ${ }^{*} P<0.05$ in $t$ test. (D and E) Cell size in gWAT(D) and iWAT (E) pads at 32 weeks of age. Data are presented as mean \pm SEM. $n=3$ /group. ${ }^{*} P<0.05$ in $t$ test.

ing either chow feeding or HFD feeding period (Figure 7C), while feeding efficiency in MeA-ER $\alpha$-KO mice was significantly increased most of the time during days 2-22 compared with control mice (Figure 7D). Importantly, chow-fed MeA-ER $\alpha$-KO mice showed significant reductions in physical activities, especially during the dark cycle (Figure 7, E and F). Finally, body temperature was comparable between the 2 groups (Figure 7, G and H). Thus, these data indicate that ER $\alpha$ in the MeA of male mice is required to prevent body weight gain, primarily through stimulating physical activity.

We also examined the physiological functions of the smaller ER $\alpha$ population in the PVN by stereotaxically injecting AAV-CreGFP into the PVN (bilaterally) of 12-week-old male $E s r 1^{f / / l}$ mice. This process selectively deleted Esr 1 in the PVN during adulthood (PVN-ER $\alpha$-KO mice; see Supplemental Figure 7A for validation). Compared to control mice, PVN-ER $\alpha$-KO mice showed comparable body weight when fed chow; these mutant mice showed a modest and slow body weight gain during HFD feeding and only became significantly heavier than control mice on day 22 (Supplemental Figure 7B). While no differences in food intake were observed during chow feeding, food intake during days 20-22 of HFD feeding was significantly higher in PVN-ER $\alpha$-KO mice than in control mice (Supplemental Figure 7C). No difference in feeding efficiency was observed during the entire period (Supplemental Figure 7D). Importantly, loss of PVN ER $\alpha$ did not affect physical activity or body temperature (Supplemental Figure 7, E-H). Thus, these data indicate that, unlike the deletion of Esr1 in the MeA, loss of PVN ER $\alpha$ caused modest body weight gain during HFD feeding, presumably due to increased food intake.

Overexpression of ER $\alpha$ in the MeA partially prevents DIO in males. To further confirm the role of MeA ER $\alpha$ signals in preventing devel- opment of DIO, we tested if overexpression of ER $\alpha$ selectively in the MeA can prevent body weight gain induced by HFD feeding. To this end, we generated a Rosa26-loxP-STOP-loxP-hESR1-flag (herein referred to as LSLhER $\alpha$ ) mouse line (Figure 8A), which allows overexpression of human ER $\alpha$ in Cre-expressing cells. Male LSLhER $\alpha$ mice received stereotaxic injections of AAV-CreGFP bilaterally into the MeA to overexpress ER $\alpha$ only in the MeA (producing MeA-ER $\alpha$-OE mice). Male WT mice receiving AAVCre-GFP injections or male LSLhER $\alpha$ mice receiving AAV-GFP injections were used as controls. Post hoc visualization of GFP was used to confirm accurate targeting to the MeA in each mouse (Figure 8B). Colocalization of GFP and flag immunoreactivity (Figure $8 \mathrm{C}$ ) validated that the $\mathrm{hER} \alpha$-flag transgene was exclusively expressed in Cre-expressing cells. We showed that body weight gain in HFD-fed MeA-ER $\alpha$-OE mice was significantly attenuated, compared with HFD-fed control mice (Figure 8D). Consistently, fat mass gain tended to decrease in MeA-ER $\alpha-\mathrm{OE}$ mice (Figure $8 \mathrm{E})$. Together, these data indicate that enhanced $\mathrm{ER} \alpha$ signals in the MeA can partially prevent DIO in male mice.

An ERa agonist activates MeA SIM1 neurons. In order to explore the cellular mechanisms for ER $\alpha$ actions in MeA SIM1 neurons, we examined effects of a highly selective ER $\alpha$ agonist, propyl pyrazole triol (PPT) (26), on the neural activities of MeA SIM1 neurons. To this end, we crossed the Rosa26-tdTOMATO allele onto Sim1-Cre mice or SIM1-ER $\alpha$-KO mice. These crosses generated control (Sim1-Cre Rosa26-tdTOMATO) and SIM1-ER $\alpha$-KO mice with TOMATO selectively expressed in SIM1 neurons. We used these mice to record the electrophysiological responses to PPT (100 nM, $50 \mathrm{~ms}$ puff) in identified MeA SIM1 neurons (Figure 9, A and B). We recorded a total of 22 neurons in control mice and found that PPT caused rapid 
A

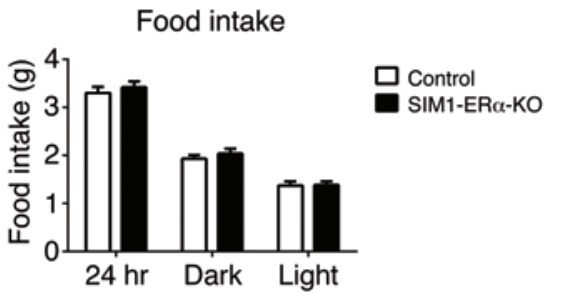

C

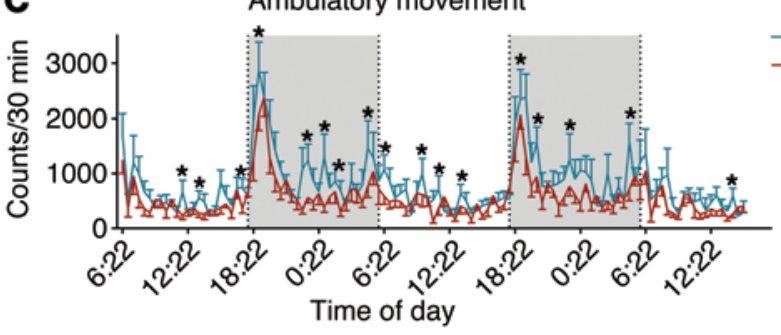

E

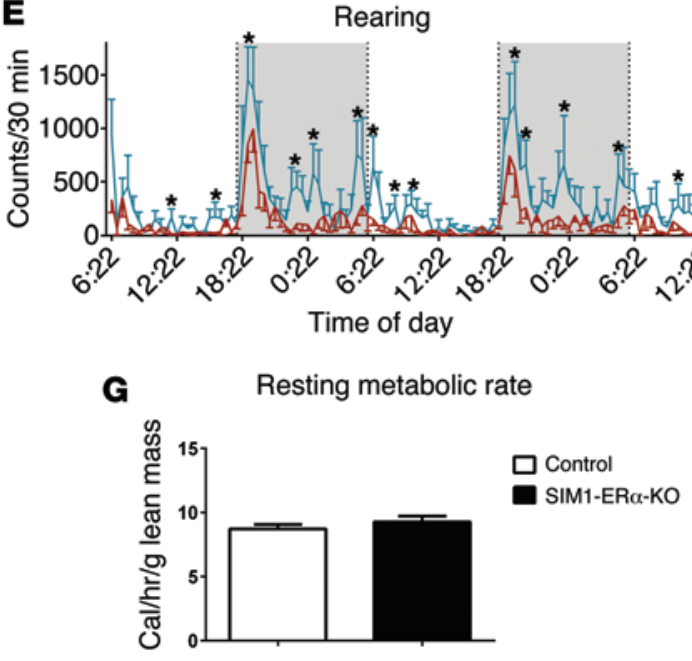

B

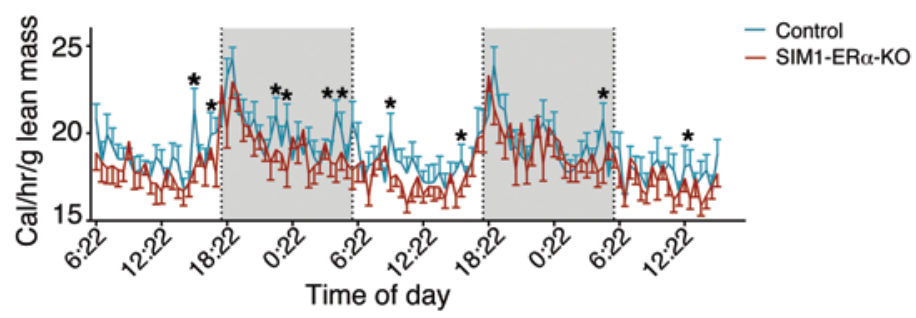

Energy expenditure
D Ambulatory movement

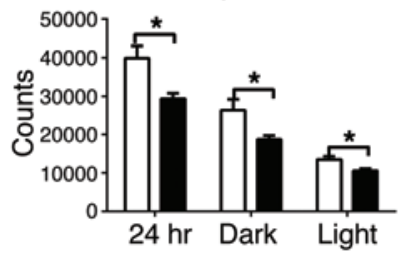

F

- SIM1-ER $\alpha-K O$

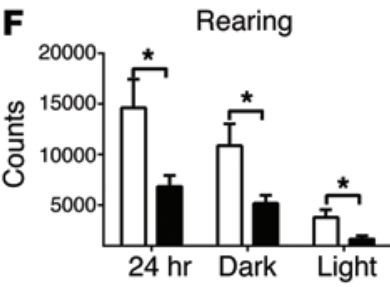

BAT thermogenic genes

$\square$ Control

SIM1-ER $\alpha-K O$

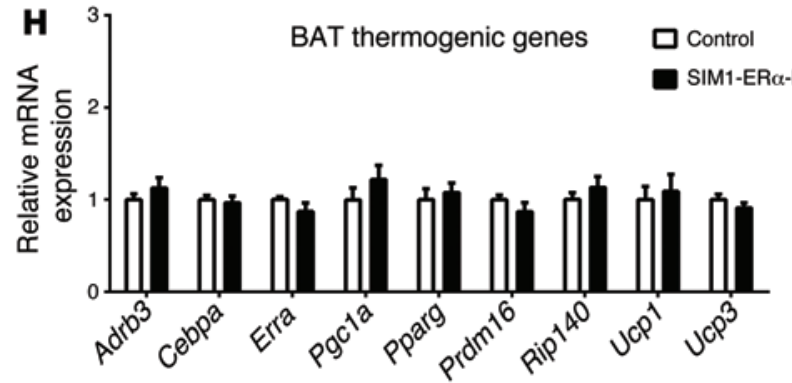

Figure 5. Metabolic profile of chow-fed male SIM1-ER $\alpha$-KO mice. (A) Food intake during 24 hour, dark, or light cycles. (B) Energy expenditure. (C and D) Ambulatory movement. (E and F) Rearing activity. (G) Resting metabolic rate. Note that mice had comparable body weight (control: $36.9 \pm 0.8$ vs. SIM1-ER $\alpha-K O: 38.0 \pm 0.9, P>0.05$ ) and lean mass (control: $24.3 \pm 0.6$ vs. SIM1-ER $\alpha-K O: 24.0 \pm 0.3, P>0.05$ ) at the time of the CLAMS study. Data are presented as mean \pm SEM. $n=12$ /group. ${ }^{*} P<0.05$ in $t$ test. $(\mathbf{H})$ mRNA levels of thermogenic genes in BAT at 32 weeks of age. Data are presented as mean \pm SEM. $n=8$ /group. ${ }^{*} P<0.05$ in $t$ test.

depolarization from rest in 12 of 22 SIM1 neurons $(2.58 \pm 0.45 \mathrm{mV}$; $n=12$; Figure 9, C, G, and I). To block action potential-dependent presynaptic activity from afferent neurons (27), tetrodotoxin (TTX, $1 \mu \mathrm{M})$ was added to the bath solution after identifying responsive SIM1 neurons that depolarized to PPT. In the presence of TTX, PPT (100 nM) application depolarized all tested SIM1 neurons (3.16 $\pm 0.96 \mathrm{mV} ; n=6$; Figure 9, D, H, and I). Lack of PPT's effects in the irresponsive neurons in control mice was presumably because these neurons did not express ER $\alpha$. Supporting this possibility, we showed that the majority of MeA SIM1 neurons from SIM1-ER $\alpha$-KO mice were not depolarized by PPT puff. Indeed, only $6.06 \%$ (2 of 33) SIM1 neurons from SIM1-ER $\alpha$-KO mice responded to PPT with a depolarization $(>1 \mathrm{mV})$. These effects were significantly attenuated compared with those in control mice (Figure 9, E, G, and I). Similarly, no neurons from SIM1-ER $\alpha-\mathrm{KO}$ mice were depolarized by PPT puff in the presence of TTX (Figure 9, F, H, and I). We further characterized effects of PPT on firing rate of MeA SIM1 neurons in control and SIM1-ER $\alpha$-KO mice. In particular, 16 of 22 neurons in control mice showed spontaneous firing at the basal condition; in these 16 neurons, 9 neurons responded to PPT with increased firing rate (Figure 9J). On the other hand, only 8 of 33 neurons in SIM1$\mathrm{ER} \alpha-\mathrm{KO}$ mice showed spontaneous firing at the basal condition, and PPT puff did not significantly alter firing rate in any of these neurons (Figure 9J). Finally, we showed that the changes in resting membrane potential and firing rate in SIM1 neurons from control mice were accompanied by a significant decrease in the whole-cell input resistance (before PPT: $152.60 \pm 4.80 \mathrm{M} \Omega$; after PPT: 132.80 $\pm 10.20 \mathrm{M} \Omega ; n=5, P<0.05$ by paired $t$ test; Figure $9, \mathrm{~K}$ and $\mathrm{L}$ ). Together, these data indicate that activation of ER $\alpha$ directly stimulates SIM1 neurons in the MeA.

Selective activation of MeA SIM1 neurons acutely promotes physical activity. Given that the ER $\alpha$ agonist activates MeA SIM1 neu- 


\section{Female}

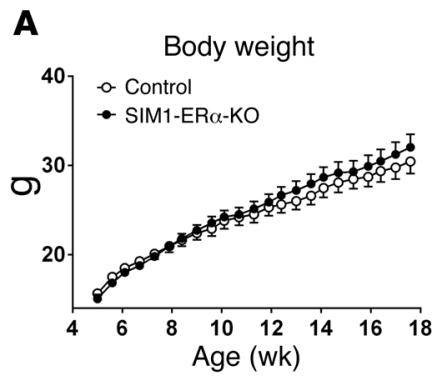

B

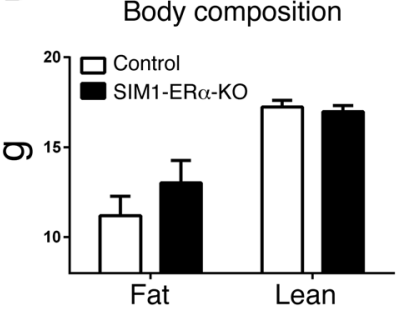

C
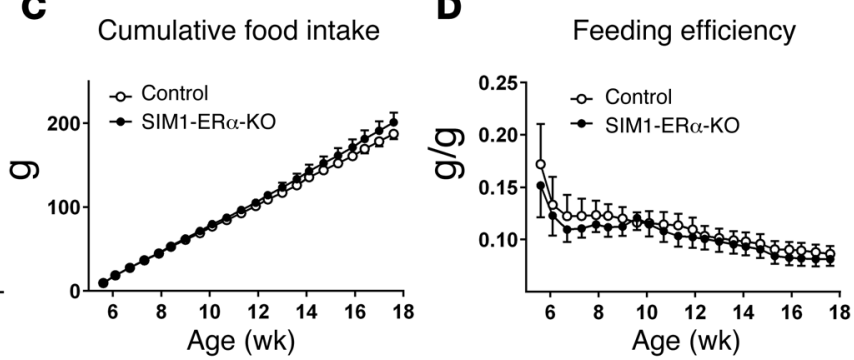

Male

E

Body weight

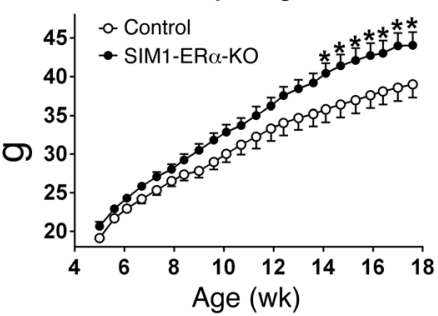

$\mathbf{F}$

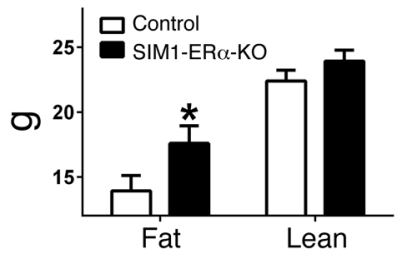

Body composition

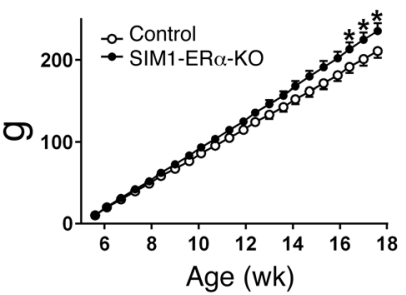

H
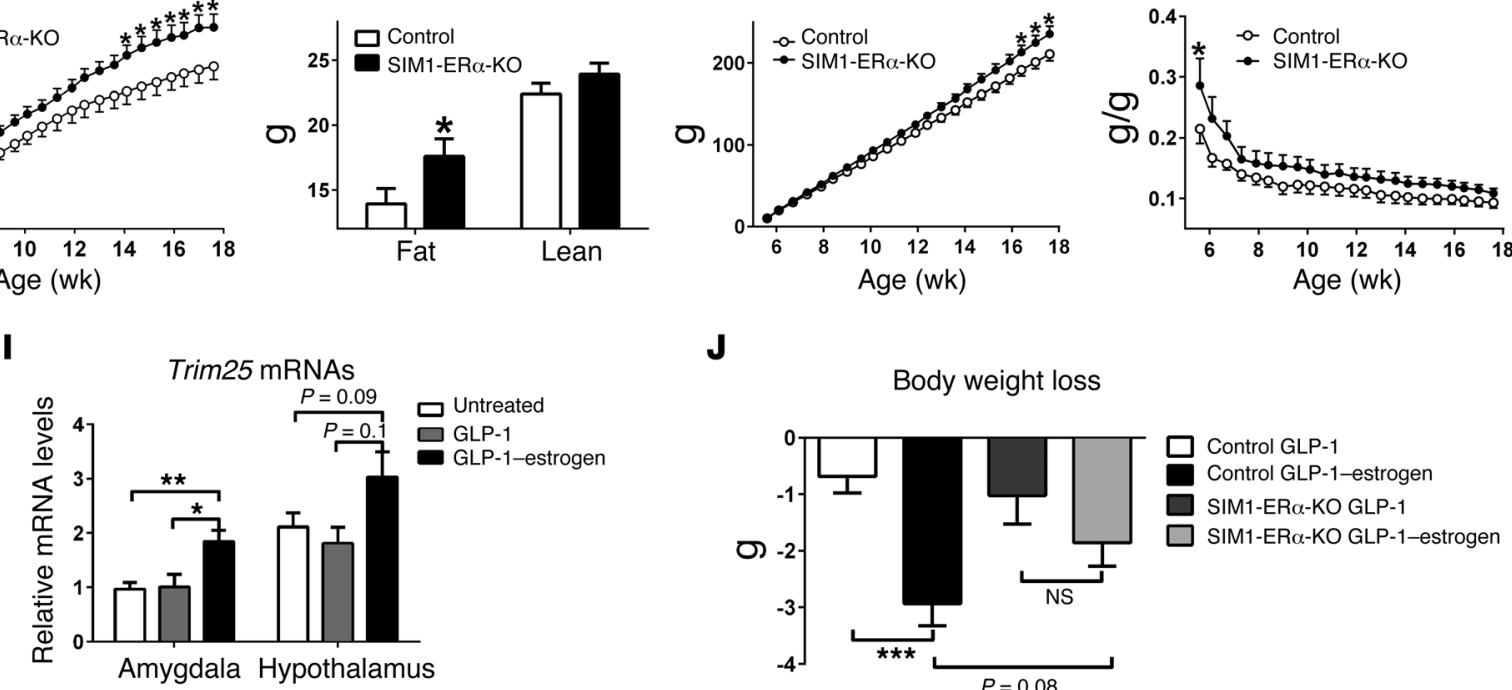

J

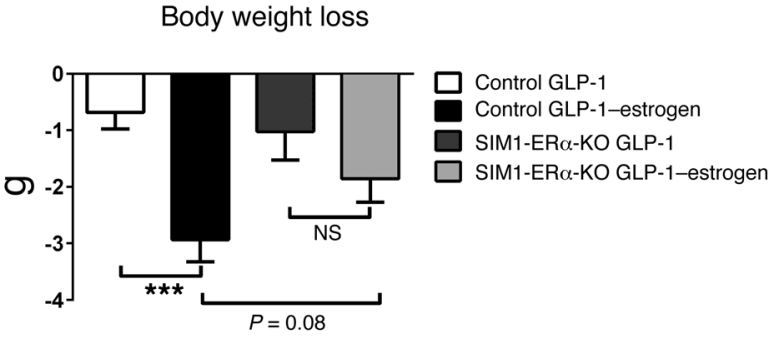

Figure 6. Metabolic profiles of HFD-fed SIM1-ER $\alpha$-KO mice. (A-D) Female control and SIM1-ER $\alpha$-KO littermates were fed with HFD for 13 weeks since weaning (4 weeks of age). Body weight (A), body fat mass and lean mass (13 weeks after HFD feeding) (B), and cumulative food intake (C) were recorded. Feeding efficiency (D) was calculated as the ratio between changes in body weight and cumulative food intake. Data are presented as mean \pm SEM. $n=18$ or 21/group. (E-H) Male control and SIM1-ER $\alpha-K O$ littermates were fed with HFD for 13 weeks since weaning (4 weeks of age). Body weight (E), body fat mass and lean mass (13 weeks after HFD feeding) (F), and cumulative food intake (G) were recorded. Feeding efficiency (H) was calculated as the ratio between changes in body weight and cumulative food intake. Data are presented as mean $\pm S E M$. $n=16 /$ group. ${ }^{*} P<0.05$ in 2-way ANOVA analysis followed by post hoc Bonferroni tests. (I) Relative Trim25 mRNA levels in the amygdala and hypothalamus of male C57BI6 mice that were untreated, or received s.c. injections of GLP-1 $(40 \mu \mathrm{g} / \mathrm{kg})$ or GLP-1-estrogen $(40 \mu \mathrm{g} / \mathrm{kg})$ for 2 hours. Data are presented as mean \pm SEM. $n=4-7 /$ group. ${ }^{*} P<0.05$ and ${ }^{* *} P<0.01$ in 1-way ANOVA analysis followed by post hoc Bonferroni tests. (J) Body weight loss in HFD-fed male control and SIM1-ER $\alpha-K O$ littermates that received s.c. injections of GLP-1 $(40 \mu \mathrm{g} / \mathrm{kg} / \mathrm{d})$ or GLP-1-estrogen ( $40 \mu \mathrm{g} / \mathrm{kg} / \mathrm{d})$ for 4 days. Data are presented as mean \pm SEM. $n=7-9 /$ group. ${ }^{* *} P<0.001$ in 2-way ANOVA analysis followed by post hoc Bonferroni tests.

rons, and that deletion of Esr1 from MeA SIM1 neurons decreases physical activity, we predict that selective activation of MeA SIM1 neurons increases physical activity. To test this, we used DREADD approach $(15,16)$ to selectively activate MeA SIM1 neurons in free-moving mice. Briefly, AAV-hM3Dq-mCherry virus was bilaterally injected into the MeA of Sim1-Cre mice (200 nl/site). Post hoc analyses validated that mCherry was exclusively expressed in the MeA of Sim1-Cre mice, as expected (Figure 10A). In addition, i.p. injections of $\mathrm{CNO}$ ( $3 \mathrm{mg} / \mathrm{kg}, 90$ minutes) induced c-FOS immunoreactivity in the MeA (Figure 10B). As negative controls, CNO-injected WT mice (without Sim1-Cre) did not show c-FOS in the MeA (Figure 10C). Similarly, no c-FOS was observed in saline-injected Sim1-Cre mice infected by DREADD virus (Figure 10D). These data validated that CNO injections selectively acti- vate MeA neurons in DREADD-infected Sim1-Cre mice. Importantly, we showed that CNO treatment $(3 \mathrm{mg} / \mathrm{kg}$, i.p.) significantly increased physical activity during the first 30 minutes after injection compared with saline treatment in the same mice (Figure 10E); no changes in body temperature (Figure 10F) and food intake (Figure 10G) were observed in CNO-treated mice compared with saline-treated mice. Notably, the increased physical activity was only transient (Figure 10H), because mice went back to sleep 1 hour after injections. Together, these data demonstrate that selective activation of MeA SIM1 neurons acutely promotes physical activity in mice.

We further explored the neurochemical identities of MeA SIM1 neurons. Interestingly, a large portion of MeA SIM1 neurons are glutamatergic, while none of MeA SIM1 neurons are GABAergic 

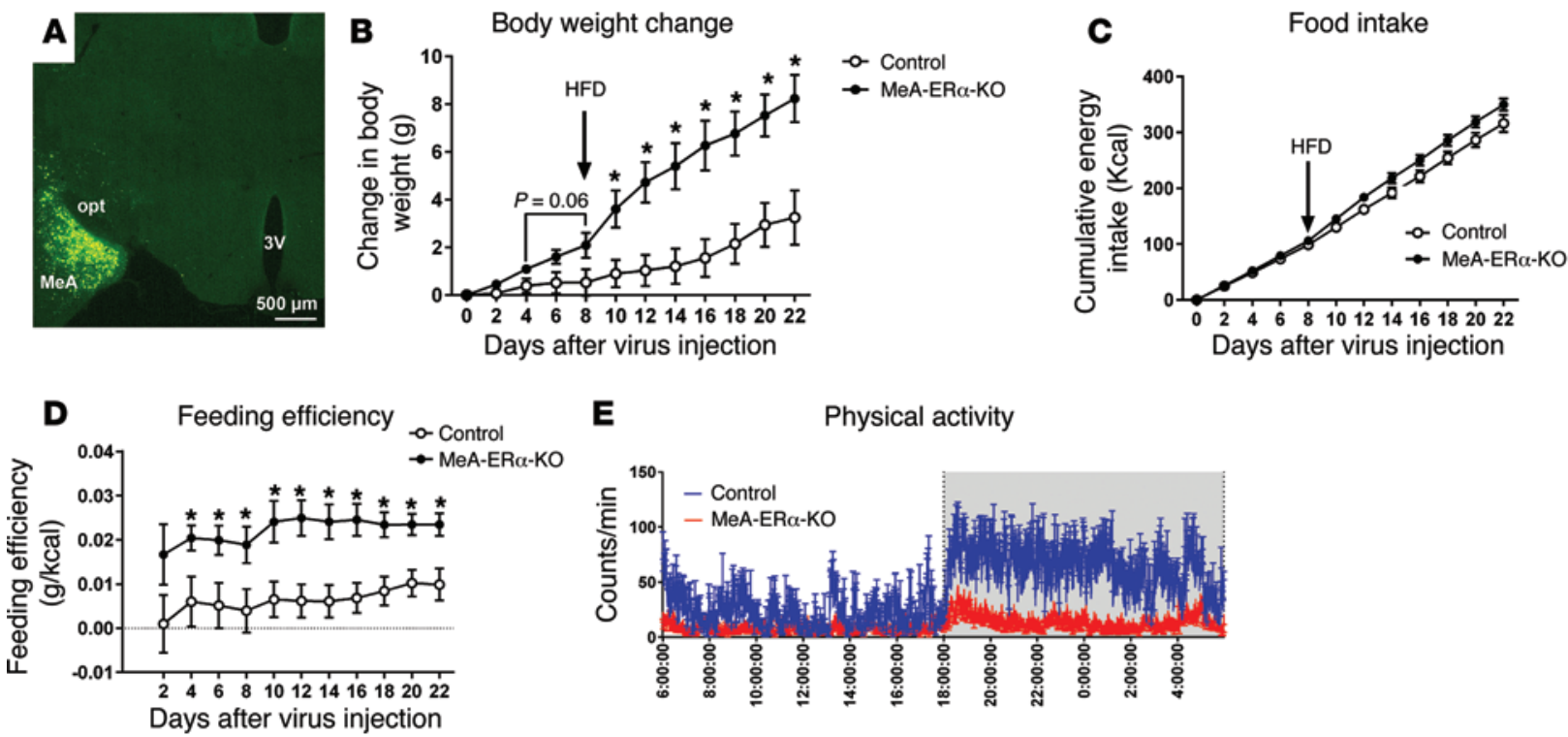

Physical activity
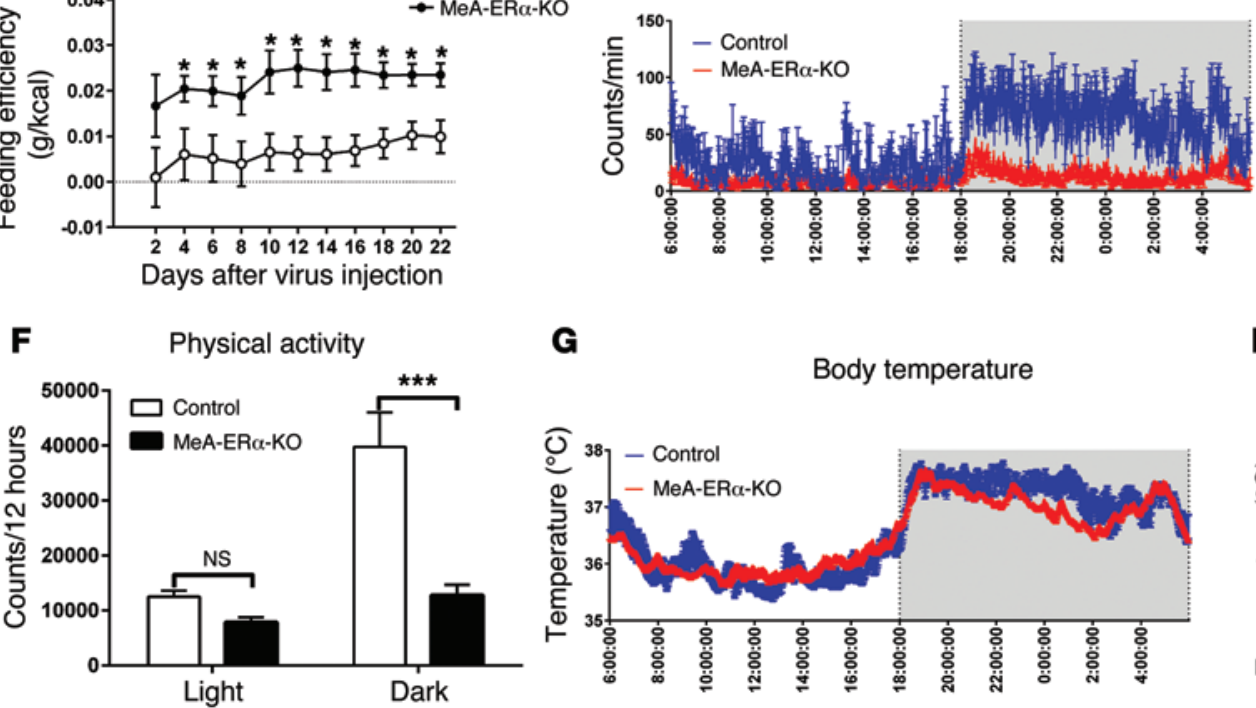

G

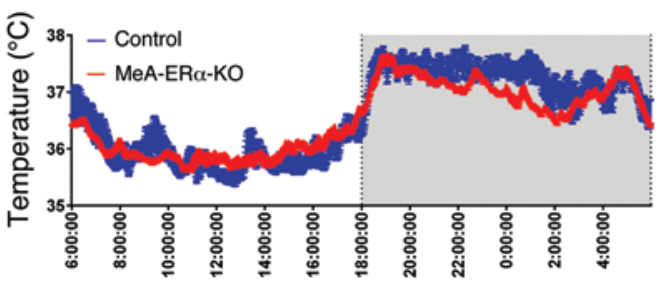

H

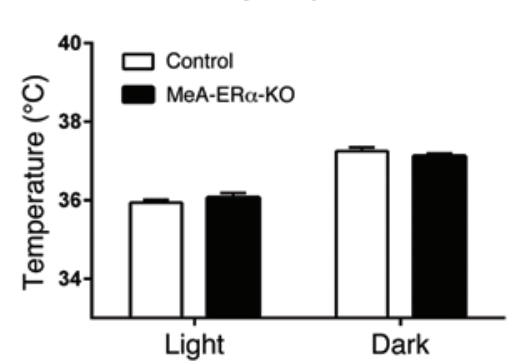

Figure 7. Metabolic profiles of MeA-ER $\alpha$-KO mice. (A) Post hoc visualization of GFP in the MeA in an Esrffl/fl mouse receiving AAV-Cre-GFP stereotaxic injections. 3V, 3rd ventricle; opt, optic tract. (B) Gains in body weight after virus injections. Note that mice were fed with regular chow for the first 7 days and then with HFD for the rest period. Data are presented as mean \pm SEM. $n=8 /$ group. ${ }^{*} P<0.05$ in 2-way ANOVA analysis followed by post hoc Bonferroni tests. (C) Cumulative food intake. (D) Feeding efficiency calculated as the ratio between changes in body weight (B) and cumulative food intake (C). Data are presented as mean \pm SEM. $n=8$ /group. ${ }^{*} P<0.05$ in 2-way ANOVA analysis followed by post hoc Bonferroni tests. (E) 24 -hour physical activity measured in chow-fed mice. (F) Sum physical activity during the light or dark cycles. Data are presented as mean \pm SEM. $n=8 / g r o u p$. ${ }^{* * *} P<0.001$ in 2-way ANOVA analysis followed by post hoc Bonferroni tests. (G) 24 -hour body temperature measured in chow-fed mice. (H) Averaged body temperature during the light or dark cycles. Data are presented as mean \pm SEM. $n=8$ /group.

(Supplemental Figures 8 and 9). In addition, to further identify the projections of MeA SIM1 neurons, we stereotaxically injected AdIN/W (28) into the MeA of Sim1-Cre mice (Supplemental Figure 10, A and B). These mice expressed wheat germ agglutinin (WGA) - an anterograde trans-synaptic tracer - specifically in MeA SIM1 neurons, which traveled along the SIM1 neural axons, passed the synapse, and labeled the downstream neurons innervated by MeA SIM1 neurons. We detected abundant WGA immunoreactivity in the red nucleus, the doral, and median Raphe nuclei (Supplemental Figure 10, C-F), suggesting that MeA SIM1 neurons project to and synapse on neurons in these regions. Future studies are warranted to determine the functional relevance of glutamate release from these MeA SIM1 neurons and to determine which SIM1 projections mediate estrogenic actions on energy homeostasis.

\section{Discussion}

MeA ERa regulates physical activity and body weight. One major finding of our study is that both male and female SIM1-ER $\alpha-\mathrm{KO}$ mice developed obesity. Systemic characterization revealed normal food intake, basal metabolic rate and BAT thermogenic gene expression in these SIM1-ER $\alpha$-KO mice, while robust reductions in physical activities were observed. Since hypoactivity was noted in young mice prior to divergence in body weight, this phenotype is unlikely to be secondary to obesity. Notably, the robust decreases in physical activity seen in SIM1-ER $\alpha$-KO mice were accompanied by only subtle reductions in overall energy expenditure. This is consistent with the notion that energy expenditure associated with physical activity only accounts for about $30 \%$ of overall energy expenditure (22).

However, these alterations in physical activity and energy expenditure are physiologically relevant, since chow-fed SIM1$\mathrm{ER} \alpha-\mathrm{KO}$ mice ultimately developed remarkable body weight gain (15\%-20\% more than control mice). Notably, the significant body weight gain was only observed at very late age, likely because the decreases in energy expenditure were subtle and need to accumulate over time to produce a detectable body weight gain. Alterna- 
A

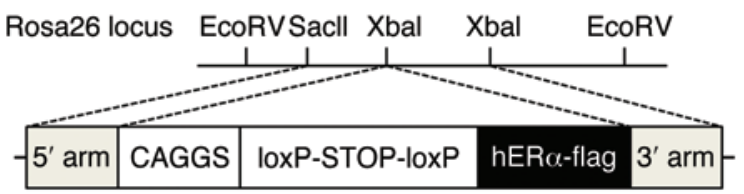

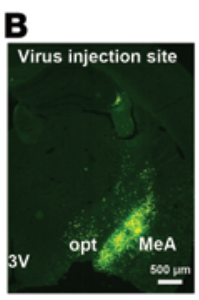

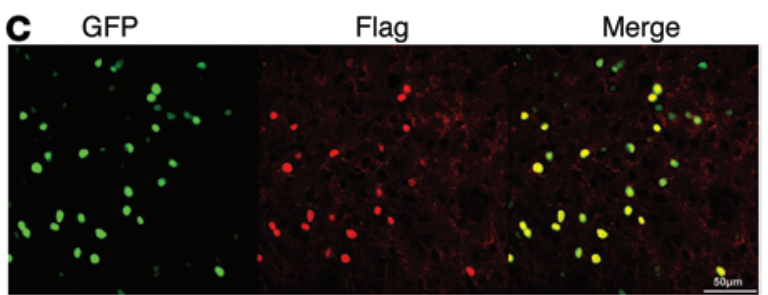

D Changes in body weight

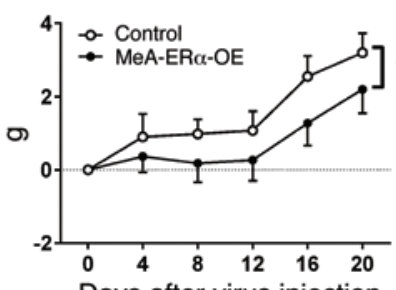

Days after virus injection

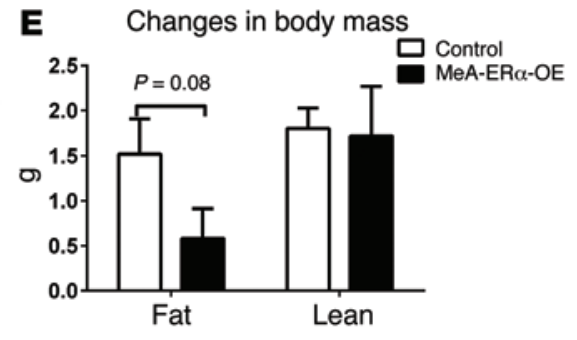

Figure 8. Metabolic profiles of MeA-ER $\alpha-O E$ mice. (A) Schematic illustration of the LSLhER $\alpha$ mouse allele. (B) Post hoc visualization of GFP in the MeA in an LSLhER $\alpha$ mouse receiving AAV-Cre-GFP stereotaxic injections. 3V, 3rd ventricle; opt, optic tract. Scale bar: $500 \mu \mathrm{m}$. (C) Colocalization of GFP and flag immunoreactivity in the MeA in an LSLhER $\alpha$ mouse receiving AAV-Cre-GFP stereotaxic injections. Scale bar: $500 \mu \mathrm{m}$. (D and E) Gains in body weight (D), and fat/lean mass (E) in HFD-fed MeA-ER $\alpha$-OE mice and control mice. Data are presented as mean \pm SEM. $n=8 /$ group. ${ }^{*} P<0.05$ in 2 -way ANOVA analysis followed by post hoc Bonferroni tests.

tively, the late-onset obesity in these mice may also suggest that $\mathrm{ER} \alpha$ actions in SIM1 neurons are more important in preventing age-associated obesity, which warrants further investigation. Nevertheless, the deficits in energy balance were exacerbated by HFD feeding, at least in males, as HFD-fed male SIM1-ER $\alpha$-KO mice developed significant body weight gain as early as 14 weeks of age.

To further determine the physiological roles of ER $\alpha$ in MeA neurons, we used stereotaxic MeA injections of AAV-Cre-GFP to delete ER $\alpha$ only in the MeA. The AAV-induced deletion produced consistent phenotypes: body weight gain associated with decreased physical activity, but normal food intake and thermogenesis. Thus, our results from $2 \mathrm{ER} \alpha$ deletion models (SIM1-induced deletion and AAV-induced deletion) provide complementary evidence to highlight the physiological role of MeA ER $\alpha$ in the regulation of physical activity and energy homeostasis.

Notably, the development of body weight gain caused by AAV-induced deletion was much faster than the late-onset obesity seen in SIM1-ER $\alpha$-KO mice. This difference may be attributed to the fact that AAV deleted more Esr1 in the MeA than in SIM1-ER $\alpha$-KO mice (about $40 \%$ of MeA ER $\alpha$ ). In addition, deletion of Esr 1 from the embryonic stage in SIM1-ER $\alpha$-KO mice may have resulted in developmental compensations that masked phenotypes. Indeed, elevated Ucp1 expression in the BAT of female SIM1-ER $\alpha$-KO mice may represent one such compensation. On the other hand, AAV injections during adulthood are expected to avoid such developmental compensations and therefore produced more profound metabolic phenotypes.

Our results indicate that $\mathrm{MeA} \mathrm{ER} \alpha$ regulates physical activity but does not affect other components of energy balance (e.g., food intake, resting metabolic rate, and thermogenesis). Interestingly, previous studies demonstrated that deletion of Esr 1 in VMH neurons decreases resting metabolic rate and BAT thermogenesis (7-9), while loss of ER $\alpha$ expressed by ARH POMC neurons leads to increased food intake in female mice (7). Furthermore, ER $\alpha$ expressed in the NTS (10), POAH $(11)$, and DRN $(11,12)$ is also implicated in the regulation of feeding in females. Collectively, these findings support a scenario where ER $\alpha$ populations in female brains are functionally segregated. Thus, ER $\alpha$ in the MeA specifically stimulates physical activity; ER $\alpha$ in the VMH stimulates resting metabolic rate and thermogenesis; and ER $\alpha$ in the ARH, NTS, $\mathrm{POAH}$, and DRN functions primarily to suppress feeding. These segregated ER $\alpha$ populations function complementarily to produce a full spectrum of estrogenic effects on female energy homeostasis.

$M e A$ ER $\alpha$ prevents DIO in males. Accumulating evidence indicates that endogenous $\mathrm{ER} \alpha$ signals also regulate body weight in males. For example, Esr1 gene deficiency results in obesity in male mice $(4,29)$ and in men $(30,31)$. In addition, administration of $17 \beta$-estradiol or its analogs reduces body weight in male mice $(3,24)$. However, the mechanisms underlying the estrogenic actions in male energy homeostasis are less documented. We previously showed that deletion of Esr 1 from the entire brain results in obesity in male mice (7). Here we demonstrate that selective deletion of Esr 1 from MeA neurons led to obesity in male mice, which further pinpoints ER $\alpha$ in MeA neurons as one of the key sites that regulate male energy balance. Notably, SIM1-induced deletion of Esr1 from male mice resulted in increased susceptibility to DIO, indicating that $\mathrm{ER} \alpha$ signals in MeA SIM1 neurons are required to defend against DIO specifically in males. The protective role of MeA ER $\alpha$ in male body weight balance was further supported by the observations that AAV-induced MeA-specific Esr1 deletion produced rapid body weight gain in male mice and that overexpression of ER $\alpha$ only in the MeA partially prevents DIO in male mice.

The major male sex hormone, testosterone, can be converted into $17 \beta$-estradiol by aromatase. Notably, both male and female aromatase KO mice develop obesity primarily due to reduced physical activity, but not to hyperphagia or decreased 

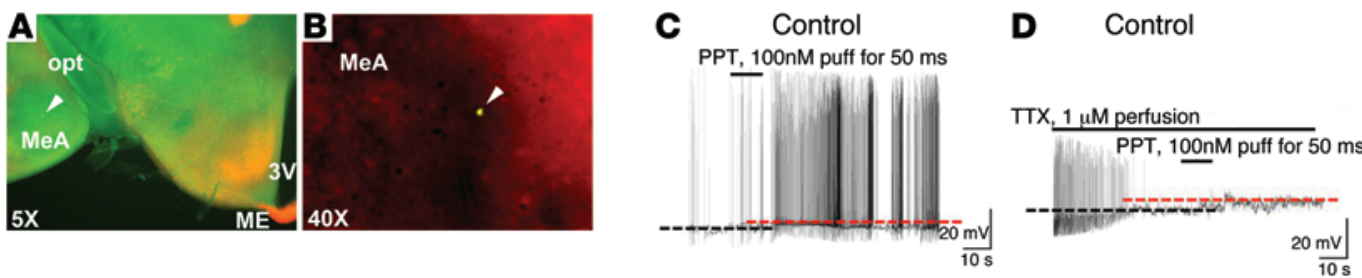

E SIM1-ER $\alpha-K O$

PPT, 100nM puff for $50 \mathrm{~ms}$

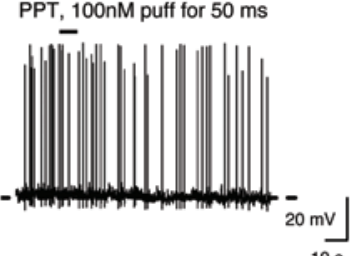

F SIM1-ER $\alpha-\mathrm{KO}$ TTX, $1 \mu \mathrm{M}$ perfusion PPT, 100nM puff for $50 \mathrm{~ms}$
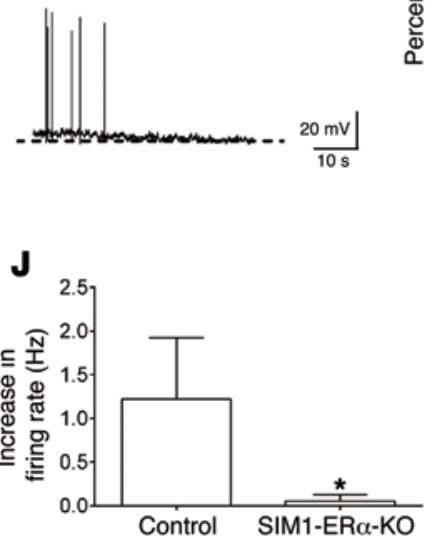

G Depolarized by PPT

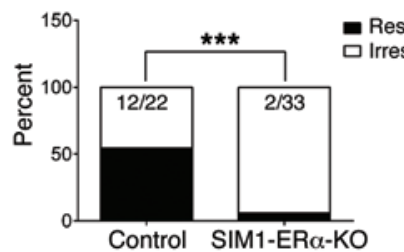

$\mathbf{K}$

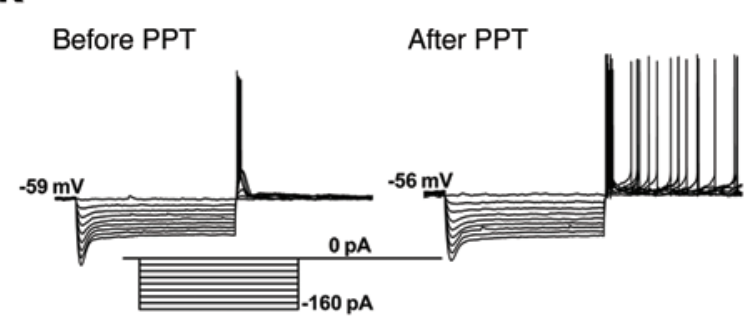

H Depolarized by PPT (TTX)
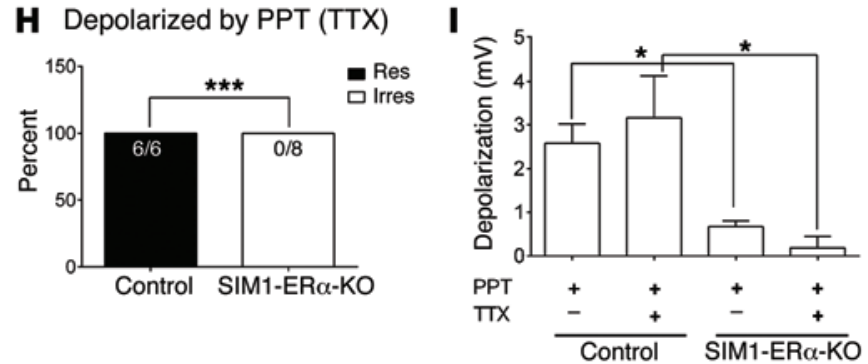

$\mathbf{L}$

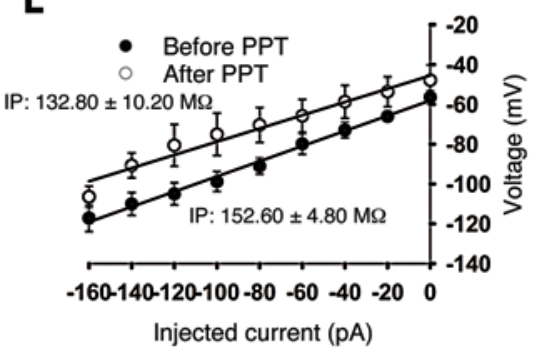

Figure 9. Effects of a selective ER $\alpha$ agonist on neural activities of MeA SIM1 neurons. (A and B) A recorded MeA SIM1 neuron, filled with the green Allex488 dye, in the brain slice from a Sim1-Cre Rosa26-tdTOMATO mouse was indicated by the arrowhead. 3V, 3rd ventricle; ME, median eminence; opt, optical tract. (C-F) Representative responses to PPT ( $100 \mathrm{nM}, 50 \mathrm{~ms}$ puff) in the absence (C and $\mathbf{E})$ or the presence of $1 \mu \mathrm{M}$ TTX (D and F) on MeA SIM1 neurons from control mice ( $\mathbf{C}$ and $\mathbf{D})$ or from SIM1-ER $\alpha-K O$ mice $(\mathbf{E}$ and $\mathbf{F})$. ( $(\mathbf{G}$ and $\mathbf{H})$ The percentage of neurons that were depolarized by PPT puff in the absence (C) or the presence (H) of $1 \mu \mathrm{M} \mathrm{TTX.}{ }^{* * *} P<0.001$ in $\chi^{2}$ test. (I) PPT-induced depolarization. Data are presented as mean \pm SEM. $n=6-33$. ${ }^{*} P<0.05$ in 2-way ANOVA analysis followed by post hoc Bonferroni tests. (J) PPT-induced increases in firing rate. Data are presented as mean \pm SEM. $n=8-9 .{ }^{*} P<0.05$ in Wilcoxon signed rank test. (K) Representative membrane voltage responses to series depolarizing steps (from -160 pA to $0 \mathrm{pA}$ in steps of $20 \mathrm{pA}$ ) for 1 second before and after PPT treatment. (L) Current-voltage curves before and after PPT treatment. Input resistance (IP) was determined by the slope of a linear regression fitted line of current-voltage curve. Results are shown as mean \pm SEM.

resting metabolic rate (32), phenotypes that almost exactly resemble mice lacking ER $\alpha$ in SIM1 neurons or in the MeA. It is worth noting that the MeA expresses abundant aromatase (33), which makes it possible that ER $\alpha$ in male MeA could be exposed to high levels of $17 \beta$-estradiol despite the lack of circulating estrogens. Interestingly, aromatase availability in the MeA has been reported to be negatively associated with body mass index in humans (34), suggesting that decreased aromatase activity, and therefore presumably decreased estrogen levels, in the MeA may contribute to development of obesity in humans. Thus, these findings raise the possibility that ER $\alpha$ in the male MeA may be a suitable target for the treatment of DIO. To test this, we used a recently developed GLP-1-estrogen conjugate, which uses GLP-1 as a carrier to deliver estrogens preferentially to GLP-1 receptor-enriched regions (24). Interestingly, the MeA expresses considerable GLP-1 receptors (25) in addition to ER $\alpha$. Furthermore, we showed that GLP-1-estrogen can deliver bioactive estrogens to the amygdala region. Importantly, we showed that GLP-1-estrogen produced less body weight loss in SIM1-ER $\alpha$-KO males than in control males. Together, these results indicate that ER $\alpha$ in the male MeA is at least one site in which GLP-1-estrogen acts to reduce body weight.
It is worth noting that while male mice lacking ER $\alpha$ in SIM1 neurons were more susceptible to DIO, female mice with the same mutation were not. The mechanisms for this sexual dimorphism are not clear. Interestingly, there appears to be more ER $\alpha$-expressing SIM1 neurons in male MeA than in female MeA. Thus, male mutants lose more ER $\alpha$ than female mutants, which may have accounted for the stronger body weight phenotype in HFD-fed male mutants. Alternatively, stronger redundant pathways may exist in female brains than in male brains, which may have prevented DIO in female mutants. Supporting this latter possibility, multiple ER $\alpha$ sites in female brains (e.g., ARH POMC neurons and VMH neurons) have been implicated in the regulation of energy balance (7), while ER $\alpha$ in MeA SIM1 neurons is the only population identified so far in male brains that regulates body weight homeostasis.

Rapid actions of ER $\alpha$ in MeA SIM1 neurons. The cellular mechanisms by which MeA ER $\alpha$ regulates physical activity are presumably through stimulation of neural activities of MeA neurons, which in turn promote physical activity. First, we showed that puff applications (50 ms) of the highly selective ER $\alpha$ agonist (PPT) rapidly activates MeA SIM1 neurons. Notably, the PPT-induced depolarization was preserved in the presence of TTX. Further- 

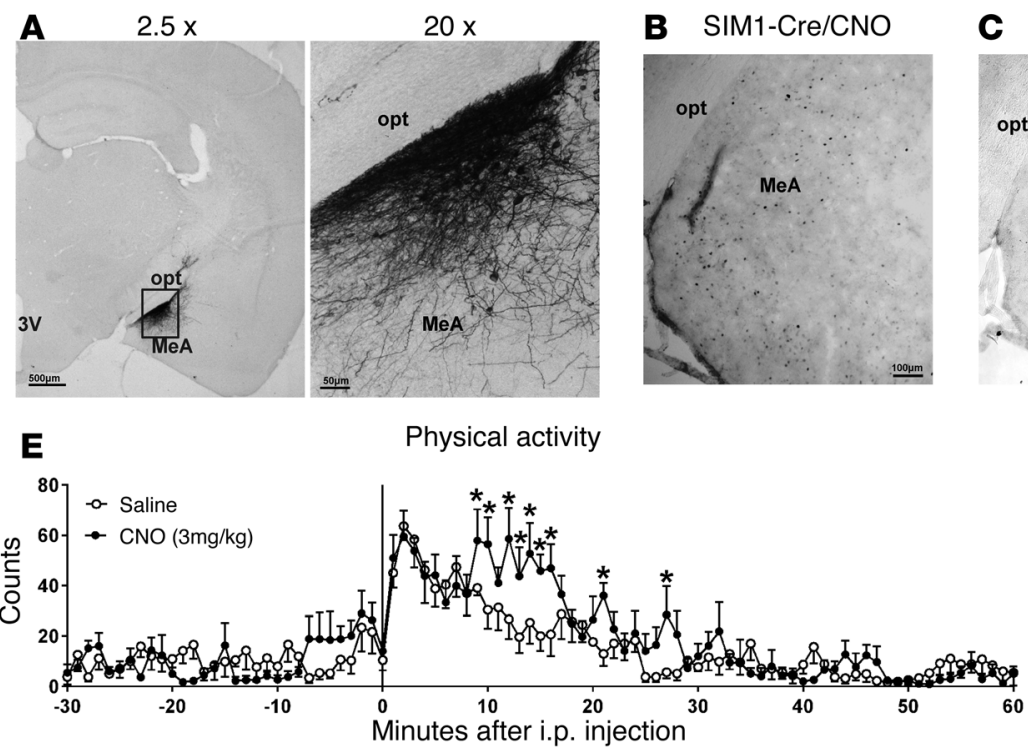

$\mathbf{F}$

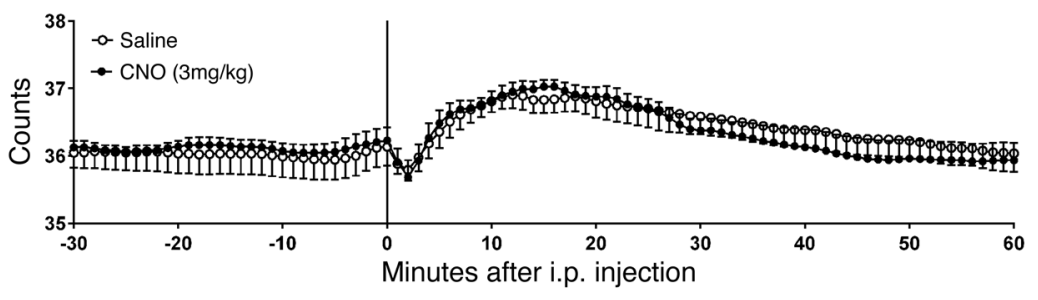

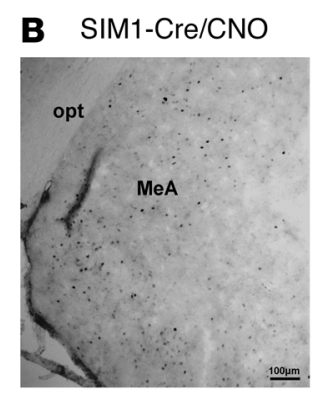
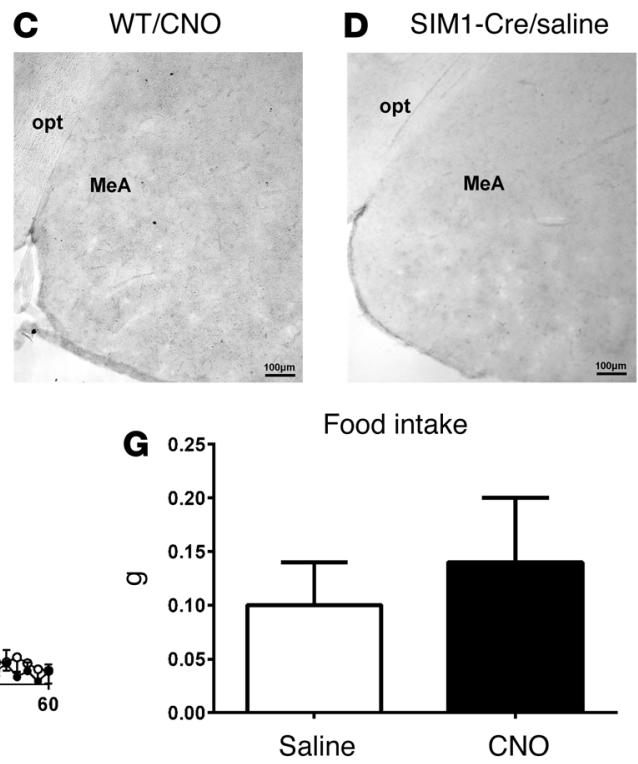

H

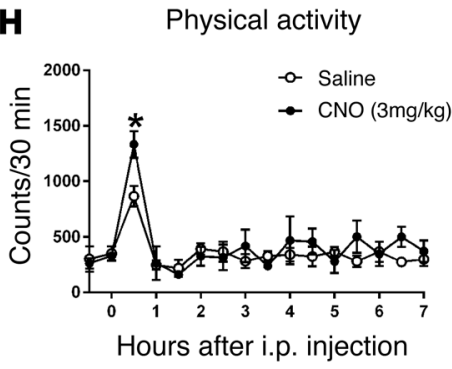

Figure 10. Effects of selective activation of MeA SIM1 neurons. (A) mCherry immunoreactivity in the MeA of Sim1-Cre mice receiving AAV-hM3DqmCherry infection. (B) c-FOS immunoreactivity in Sim1-Cre mice receiving AAV-hM3Dq-mCherry infection and CNO injection (3 mg/kg, i.p.). (C) c-FOS immunoreactivity in WT mice receiving AAV-hM3Dq-mCherry infection and CNO injection (3 mg/kg, i.p.). (D) c-FOS immunoreactivity in Sim1-Cre mice receiving AAV-hM3Dq-mCherry infection and saline injection. 3V, 3rd ventricle; opt, optic tract. (E-H) AAV-hM3Dq-mCherry-infected Sim1-Cre mice received i.p. injections of saline or $\mathrm{CNO}(3 \mathrm{mg} / \mathrm{kg})$ at 9 am. Physical activity $(\mathbf{E})$ and body temperature (F) were measured. (G) Food intake during 2 hours after i.p. injections in mice. (H) Physical activity within 30-minute bin was monitored for up to 7 hours after i.p. injections in mice. Data are presented as mean \pm SEM. $n=6$ /group. ${ }^{*} P<0.05$ in $t$ tests.

more, deletion of Esr1 blocked PPT's effects in the majority of SIM1 neurons. Thus, PPT effects are likely mediated directly via ER $\alpha$ expressed by MeA SIM1 neurons. These findings are consistent with earlier observations that estrogens directly activate other neural populations $(12,35,36)$. Furthermore, we tested the physiological functions of MeA SIM1 neural activation via the DREADD approach. Interestingly, selective activation of MeA SIM1 neurons rapidly promotes physical activity in free-moving mice without affecting their body temperature and feeding behavior. These results are consistent with observations that loss of ER $\alpha$ in MeA neurons leads to robust reductions in physical activity but normal body temperature and food intake. Therefore, our results support a model that estrogens act upon ER $\alpha$ to rapidly activate MeA SIM1 neurons, which in turn promote physical activity. Notably, the increases in physical activity were only transient because mice went back to sleep. We interpreted these findings to suggest that while activation of MeA SIM1 does promote physical activity, these MeA SIM1 neurons do not regulate arousal, and therefore activation of these neurons alone is not sufficient to override normal circadian rhythms and promote long-term acti- vation of physical activity. Interestingly, we further demonstrated that most of SIM1 neurons in the MeA are glutamatergic but not GABAergic. It was recently reported that activation of glutamatergic neurons in the posterior dorsal subdivision of MeA acutely promotes self-grooming activity and inhibits aggressive behavior, while activation of GABAergic neurons in the same region triggers aggressive behavior (37). Although these social behaviors were not directly measured in our model, we speculate that the increased physical activities we observed could be an important component of the complex behaviors governed by these MeA neurons.

In summary, our results indicate that ER $\alpha$ in the MeA mediates estrogenic actions to stimulate physical activity and therefore promotes energy expenditure and prevents obesity. This extrahypothalamic ER $\alpha$ population constitutes an important component of the estrogen-responsive neural networks in female brains and coordinates with ER $\alpha$ populations in other brain regions to mediate the full spectrum of body weight-lowering effects of estrogens. Strikingly, in males, the functions of MeA ER $\alpha$ in physical activity and in body weight homeostasis are preserved and even more important, especially in the context of HFD feeding. 
Notably, endogenous $\mathrm{ER} \alpha$ signals in the male MeA are required to defend DIO; enhanced ER $\alpha$ signals in this region, either by genetic overexpression or by pharmacological agents, provide weight loss benefits during HFD feeding. These results indicate that MeA ER $\alpha$ is a potential therapeutic target for treatment of obesity.

\section{Methods}

Mice. All mouse strains were backcrossed onto C57BL/6 background for more than 12 generations. Sim1-Cre transgene (20) was bred onto $E s r 1^{f / f l}$ mice (21) to generate Sim1-Cre Esr $1^{f / f l}$ and Esr $1^{f / f l}$ littermates, which were referred to as SIM1-ER $\alpha-\mathrm{KO}$ and control mice, respectively. At 4 weeks of age, mice were either fed with normal chow diet (catalog 2916, Harlan Teklad) or 60\% HFD (catalog D12492, Research Diets Inc.). These mice were used to characterize the metabolic profile, as described below. In parallel, Sim1-Cre Esr1 ${ }^{f l /+}$ mice were crossed with Esr $1^{f /+}$ Rosa26-GFP mice to generate Esr $1^{f / / t}$ Sim1-Cre Rosa26-GFP (SIM1-ER $\alpha$-KO-GFP) mice and Esr1/++ Sim1-Cre Rosa26-GFP (control) mice. These mice were used for histology validation. In addition, $E s r 1^{f l+}$ mice were crossed with $E s r 1^{f /+}$ to generate $E s r 1^{f / f l}$ mice and $E s r 1^{+/+}$(control) mice, which received AAV-Cre-GFP stereotaxic injections into the MeA.

In addition, we generated LSLhER $\alpha$ allele using similar approaches as described previously (38). In brief, human ESR1 cDNA (courtesy of Pierre Chambon, the Institut de Génétique et de Biologie Moléculaire et Cellulaire (IGBMC), Illkirch, CU. de Strasbourg, France) was subcloned into the pRosa26-CAGGS-loxP-STOP-loxP-Luciferase vector (39), replacing the luciferase cDNA. The resulting pRosa26-CAGGSloxP-STOP-loxP-hESR1 targeting construct was utilized to create the $C A G-S-h E S R 1$ allele at the Rosa26 locus of AB2.2 embryonic stem cells. The minigene in this allele constitutively expresses human ER $\alpha$ with an N-terminal Myc-FLAG tandem tag after removal of the STOP cassette. LSLhER $\alpha$ heterozygous mice were crossed with C57BL/6 mice to produce male LSLhER $\alpha$ heterozygous mice and WT littermates. These mice were used for overexpression study.

Sim1-Cre transgenic mice were crossed with C57BL/6 mice to produce male Sim1-Cre transgenic mice and WT littermates, which were used for DREADD study. In addition, some Sim1-Cre transgenic mice were crossed with Rosa26-tdTOMATO mice to generate Sim1-Cre Rosa26-tdTOMATO mice, which were used for electrophysiological recordings.

Colocalization of SIM1 and ER $\alpha$. Female or male SIM1-ER $\alpha-\mathrm{KO}-$ GFP and Sim1-Cre Rosa26-GFP mice were perfused with 10\% formalin. To avoid influence of estrous cycles on expression pattern, female mice were all perfused at diestrus. Brain sections were cut at $25 \mu \mathrm{m}$ (1:5 series), and the sections were incubated in the primary rabbit anti-ER $\alpha$ antibody (1:10,000; catalog C1355, Millipore) overnight, followed by donkey anti-rabbit AlexaFluor594 (1:500; catalog A21207, Invitrogen) for 1.5 hours. Then, the sections were incubated in primary chicken anti-GFP antibody (1:5,000; catalog GFP-1020, Aves Labs Inc.) overnight, followed by the goat anti-chicken AlexaFluor 488 (1:250; catalog A11039, Invitrogen) for 1.5 hours. For each mouse, the numbers of SIM1 neurons, ER $\alpha$ neurons, and double-labeled neurons were counted in all coronal brain sections that contain the MeA, POAH, PVN, PH, LOT, SON, and PAG. Data were collected and averaged from 3 mice per genotype $\mathrm{X}$ sex.

Body weight, body composition, food intake, and feeding efficiency. Male and female SIM1-ER $\alpha-K O$ and control littermates were weaned on chow or HFD. Body weight was monitored twice a week until the end of the study. Body composition was determined using quantitative magnetic resonance (QMR) at the time as indicated in the figure legends. HFD-fed cohorts were singly housed in order to measure food intake in home cages. For the single-housed HFD-fed mice, energy expenditure was estimated by calculating feeding efficiency (changes in body weight/cumulative food intake).

Adipose tissue analyses. At the end of body weight monitoring, chow-fed male and female SIM1-ER $\alpha$-KO and control littermates were deeply anesthetized and decapitated. The gWAT and the iWAT were isolated and weighted. One piece of each fat pad was fixed with $10 \%$ formalin overnight and then stored in $50 \%$ ethanol. The fixed fat pads were sent to Cellular and Molecular Morphology Core at Texas Medical Center, where the tissues were subjected to H\&E staining. To determine adipocyte size in WAT tissues, pictures of the H\&E staining were obtained under the red fluorescence channel using a fluorescence microscope and analyzed using Image J software. Eight hundred to 1,000 cells from each sample were included in the analysis. Data from 3 mice were collected and averaged for each group.

Quantitative PCR. After male and female SIM1-ER $\alpha-K O$ and control littermates were decapitated, interscapular BAT depot, hypothalami, and amygdala were quickly microdissected and stored at $-80^{\circ} \mathrm{C}$. Quantitative PCR (qPCR) was performed as described previously (40). Primer sequences were listed in Supplemental Table 2.

Serum analyses. After male and female SIM1-ER $\alpha-\mathrm{KO}$ and control littermates were decapitated, trunk blood was collected and centrifuged to obtain serum. Serum samples were sent to the Hormone Assay \& Analytical Services Core at Vanderbilt University to measure $17 \beta$-estradiol, testosterone, T3/T4, and norepinephrine/epinephrine. Serum leptin was measured using ELISA kits (catalog 90030, Crystal Chem Inc.).

CLAMS metabolic chambers. Food intake, physical activity and energy expenditure were monitored using CLAMS (Columbus Instruments). Briefly, male or female chow-fed SIM1-ER $\alpha$-KO and control mice were housed individually in CLAMS cages. After adaptation for 5 days, ambulatory movement, rearing, physical activity, $\mathrm{O}_{2}$ consumption, $\mathrm{CO}_{2}$ production, and heat production were monitored for 2 days. We performed these experiments in young mice with matched body weight, fat mass, and lean mass; energy expenditure was normalized by lean mass (41). To assess the resting metabolic rate, diet was removed at 8 am (2 hours after the onset of light cycle), and returned to the chambers at $6 \mathrm{pm}$ (the onset of dark cycle). The lowest energy expenditure value during the light cycle was used to estimate resting metabolic rate.

Effects of GLP-1-estrogen. Male C57Bl6 mice (16 weeks of age) received s.c. injections of GLP-1 $(40 \mu \mathrm{g} / \mathrm{kg})$ or GLP-1-estrogen $(40 \mu \mathrm{g} / \mathrm{kg})$. Two hours after injections, mice were decapitated, and hypothalami and amygdala were quickly microdissected and stored at $-80^{\circ} \mathrm{C}$. mRNA levels of Trim 25 were measured with qPCR as described above.

To test the antiobesity effects of GLP-1 and GLP-1-estrogen, male SIM1-ER $\alpha-K O$ and control were fed on HFD to induce DIO. Mice were randomly divided into 2 groups to receive daily s.c. injections of GLP-1 $(40 \mu \mathrm{g} / \mathrm{kg} / \mathrm{d})$ or GLP-1-estrogen $(40 \mu \mathrm{g} / \mathrm{kg} / \mathrm{d})$ for 4 days. Body weight was monitored daily. Note that average body weight was comparable among 4 groups prior to injections.

Reproductive functions. A separate cohort of SIM1-ER $\alpha-K O$ and control littermates was used to assess their reproductive functions, as we did before (7). 
Behavioral tests for anxiety. An independent cohort of SIM1-ER $\alpha-\mathrm{KO}$ and control littermates (both males and females, 8 weeks of age) were subjected to the open field tests, the light-dark tests, and the elevated plus maze tests, as we did before (12). Note that all the behavioral tests were performed at around $9 \mathrm{am}-10$ am during the light cycles.

Deletion of Esr1 in the MeA or in the PVN. Male Esr ${ }^{f l / f l}$ mice were anesthetized by isoflurane and received bilateral stereotaxic injections of AAV-Cre-GFP (AAV9.CMV.HI.GFP-Cre.WPRE.SV40, Penn Vector Core at University of Pennsylvania, Philadelphia, PA) into the MeA or the PVN (200 nl/side), and these mice were referred to as MeA-ER $\alpha$ KO or PVN-ER $\alpha$-KO. Control mice included $E s r 1^{+/+}$littermates that received the same AAV-Cre-GFP infection or $E s r 1^{f l / f l}$ mice that receive AAV-GFP in the same brain region. The coordinates for the MeA were $1.7 \mathrm{~mm}$ posterior and $2.5 \mathrm{~mm}$ lateral to the bregma, and $5 \mathrm{~mm}$ ventral to the dura; the coordinates for the PVN were $0.58 \mathrm{~mm}$ anterior and $0.25 \mathrm{~mm}$ lateral to the bregma, and $4.75 \mathrm{~mm}$ ventral to the dura. Under the same anesthesia, a telemetric Mini Mitter probe ER-4000 (E-mitter; Philips Respironics) was implanted into the abdominal cavity. These mice were fed chow for the first 7 days after the surgery and then fed with HFD from day 8-22. Body weight and food intake were measured every 2 days after virus injections. During days 3-7, mouse cages were put on top of the ER-4000 Receivers (Philips Respironics) (42) to measure physical activity and body temperature for 5 consecutive days, and data were averaged for each mouse. At the end of the study, each mouse was perfused and brain sections were collected as described above. GFP was detected to validate accurate injections into the MeA or the PVN. Only those with accurate injections in both sides of MeA or PVN were included in data analyses.

Overexpression of ER $\alpha$ in the MeA. Male LSLhER $\alpha$ heterozygous mice (at 8-10 weeks of age) were anesthetized and received bilateral stereotaxic injections of AAV-Cre-GFP into the MeA (200 nl/side) as described above, and these mice were referred to as MeA-ER $\alpha-\mathrm{OE}$ mice. Similarly, some male LSLhER $\alpha$ heterozygous mice received AAV-GFP injections, and male WT mice received AAV-Cre-GFP injections to serve as controls. Mice were then fed on $60 \%$ HFD. Body weight was measured every 2 days after virus injections, and body composition was measured by QMR before and 20 days after virus injections.

At the end of the study, each mouse was perfused and brain sections were collected as described above. IHC for flag was used to validate the overexpression of ER $\alpha$-flag in the MeA. Briefly, the brain sections were incubated overnight with mouse anti-flag monoclonal antibody (1:10,000; Sigma-Aldrich), followed by donkey anti-mouse AlexaFluor594 (1:500; Invitrogen) for 1.5 hours. GFP was detected to validate accurate injections into the MeA. Flag immunoreactivity was detected to validate overexpression of hER $\alpha$-flag exclusively in Cre-expressing (GFP) cells. Only those with accurate injections in both MeA sides were included in data analyses.

Whole-cell patch clamp. Whole-cell patch clamp recordings were performed on TOMATO-labeled SIM1 neurons in the acute MeA slices from Sim1-Cre Rosa26-tdTOMATO mice and Esr1 fl/fl Sim1-Cre Rosa26-tdTOMATO mice. Six- to 12-week-old mice were deeply anesthetized with isoflurane and transcardially perfused $(12,43,44)$ with a modified ice-cold sucrose-based cutting solution (adjusted to $\mathrm{pH}$ 7.3) containing (in $\mathrm{mM}$ ) $10 \mathrm{NaCl}, 25 \mathrm{NaHCO}_{3}, 195$ sucrose, 5 glucose, $2.5 \mathrm{KCl}, 1.25 \mathrm{NaH}_{2} \mathrm{PO}_{4}, 2 \mathrm{Na}$ pyruvate, $0.5 \mathrm{CaCl}_{2}$, and $7 \mathrm{MgCl}_{2}$ bubbled continuously with $95 \% \mathrm{O}_{2}$ and $5 \% \mathrm{CO}_{2}(45)$. The mice were then decapitated, and the entire brain was removed and immediately submerged in ice-cold sucrose-based cutting solution. Coronal sections containing the MeA $(270 \mu \mathrm{m})$ were cut with a Microm HM 650 V vibratome (Thermo Fisher Scientific Inc.). The slices were recovered for at least 1 hour at $34^{\circ} \mathrm{C}$ in artificial cerebrospinal fluid (aCSF, adjusted to pH7.3) containing (in mM) $126 \mathrm{NaCl}, 2.5 \mathrm{KCl}, 2.4 \mathrm{CaCl}_{2}, 1.2 \mathrm{NaH}_{2} \mathrm{PO}_{4}$, $1.2 \mathrm{MgCl}_{2}, 11.1$ glucose, and $21.4 \mathrm{NaHCO}_{3}(46)$ saturated with $95 \% \mathrm{O}_{2}$ and $5 \% \mathrm{CO}_{2}$ before recording.

Slices were transferred to the recording chamber and allowed to equilibrate for at least 10 minutes before recording. The slices were perfused at $34{ }^{\circ} \mathrm{C}$ in oxygenated aCSF at a flow rate of $1.8-2 \mathrm{ml} / \mathrm{min}$. tdTOMATO-positive SIM1 neurons in MeA were visualized using epifluorescence and IR-DIC imaging on an upright microscope (Eclipse FN-1, Nikon) equipped with a moveable stage (MP-285, Sutter Instrument Co.). Patch pipettes with resistances of 5-7 M $\Omega$ were filled with intracellular solution (adjusted to $\mathrm{pH}$ 7.3) containing (in $\mathrm{mM}$ ) 128 K gluconate, $10 \mathrm{KCl}, 10$ HEPES, 0.1 EGTA, $2 \mathrm{MgCl}_{2}, 0.05 \mathrm{Na}-\mathrm{GTP}$, $0.05 \mathrm{Mg}$-ATP, and 0.003 Alexa Fluor 488 fluorescent dyes (45). Recordings were made using a MultiClamp 700B amplifier (Molecular Devices), sampled using Digidata 1440A, and analyzed offline with pClamp 10.3 software (Molecular Devices). Series resistance was monitored during the recording, and the values were generally $<10$ $\mathrm{M} \Omega$ and were not compensated. The liquid junction potential (LJP) was $+12.5 \mathrm{mV}$ and was corrected after the experiment. Data would be excluded if the series resistance increased dramatically during the experiment or without overshoot for action potential.

PPT (100 nM, Sigma-Aldrich) $(26,47)$ puff application (50 ms by about 4-psi pressure ejection, Picospritzer III, Parker) and TTX $(1 \mu \mathrm{M}, \mathrm{R} \& \mathrm{D}$ system) perfusion $(4-6 \mathrm{mins}$ at $2 \mathrm{ml} / \mathrm{min})$ were selected in some experiments.

DREADD activation of MeA SIM1 neurons. The DREADD virus was provided by Bryan L. Roth (University of North Carolina, Chapel Hill, North Carolina, USA). Male Sim1-Cre mice and WT littermates (at 14-16 weeks of age) were anesthetized by isoflurane and received bilateral stereotaxic injections of AAV-hM3Dq-mCherry (AAV-hSyn-DIO-hM3D[Gq]-mCherry in AAV2; UNC Vector Core, Chapel Hill, North Carolina, USA) into the MeA ( $200 \mathrm{nl} / \mathrm{side})$. Under the same anesthesia, a telemetric E-mitter was implanted into the abdominal cavity, as described above. After a 7-day recovery, physical activity was recorded using the ER-4000 Receivers in singly housed mice in their home cages. Recordings started from 8 am and ended at $4 \mathrm{pm}$. Saline or CNO $(3 \mathrm{mg} / \mathrm{kg})$ was i.p. injected at 9 am. Food intake was measured for 2 hours after i.p. injections. After a rest for 3 days, the same mice were recorded at the same time but with different injections (CNO or saline). On day 30 after virus injections, all mice were perfused with $10 \%$ formalin. Brain sections were collected and subjected to mCherry IHC. Only those with accurate injections in both MeA sides were included in data analyses. To validate DREADD-induced activation of MeA SIM1 neurons, some Sim1-Cre mice were perfused 1 hour after an i.p. injection of CNO $(3 \mathrm{mg} / \mathrm{kg})$, and brain sections were subjected to c-fos IHC. As negative controls, c-fos immunoreactivity was also examined in WT mice receiving i.p. CNO (3 $\mathrm{mg} / \mathrm{kg}$ ) or Sim1-Cre mice receiving i.p. saline.

For m-Cherry or c-fos IHC, brain sections were first incubated in $0.3 \% \mathrm{H}_{2} \mathrm{O}_{2}$ in $\mathrm{PBS}$ for 30 minutes to abolish endogenous peroxidase activity. After several washes, sections were incubated overnight at room temperature with the primary antibodies, which were rabbit Living Colors DsRed polyclonal antibody (1:1,000; Clontech) or rab- 
bit anti-c-fos polyclonal antibody (1:100,000; Millipore). After several washes, sections were incubated with the biotinylated secondary antibody (1:1,000, anti-rabbit; Jackson ImmunoResearch Laboratories Inc.) for 1 hour. Sections were then washed and visualized by incubation with the VECTASTAI ABC kit (Vector Laboratories) according to the manufacture's instruction. Sections were washed and treated with diaminobenzidine (Sigma-Aldrich) for 5 minutes, followed by dehydration in a graded ethanol series from $50 \%-100 \%$ and a final wash in xylene.

Colocalization of SIM1 and Glutamate/GABA in the MeA. Single immunofluorescence for glutamate/GABA was used to examine colocalization of glutamate/GABA and SIM1 in Sim1-Cre Rosa26-tdTOMATO mice. Briefly, male Sim1-Cre Rosa26-tdTOMATO mice were perfused with $10 \%$ formalin. Brain sections were cut at $25 \mu \mathrm{m}$ ( 5 series), and the sections were incubated in the primary rabbit anti-glutamate antibody (1:1,000; catalog G6642, Sigma-Aldrich) or mouse anti-GABA antibody (1:1,000; catalog A0310, Sigma-Aldrich) overnight, followed by donkey anti-rabbit AlexaFluor488 (1:250; catalog A21206, Invitrogen) or goat anti-mouse AlexaFluor488 (1:250; catalog A11001, Invitrogen) for 1.5 hours.

$A d-I N / W$ anterograde tracing. In order to explore where MeA SIM1 neurons project to, 12-week-old Sim1-Cre mice were anesthetized by isofluorane and received stereotaxic injections of Ad-IN/W (28) (200 nl) into the MeA. Ad-IN/W virus was provided by Martin G. Myers Jr. (University of Michigan, Ann Arbor, Michigan, USA). In these mice, Ad-IN/W induced expression of WGA only in MeA SIM1 neurons; WGA anterogradely traveled along the SIM1 fibers, passed the synapse, and filled the downstream neurons that were innervated by SIM1 terminals. Three days after injections, mice were perfused with $10 \%$ formalin and brain sections were cut at $25 \mu \mathrm{m}$ ( 5 series). The sections were incubated at room temperature in primary goat anti-WGA antibody (1:5,000; catalog 20079, ImmunoStar Inc.) overnight, followed by the secondary donkey anti-goat Alexa Fluor 488 (1:500; catalog A-11055, Invitrogen) for 1.5 hours.
Statistics. The data are presented as mean \pm SEM. Statistical analyses were performed using GraphPad Prism to evaluate normal distribution and variations within and among groups. Methods of statistical analyses were chosen based on the design of each experiment and are indicated in figure legends. $P<0.05$ was considered to be statistically significant.

Study approval. Care of all animals and procedures were conformed to the Guide for Care and Use of Laboratory Animals of the US National Institutes of Health and were approved by the Animal Subjects Committee of Baylor College of Medicine.

\section{Acknowledgments}

This work was supported by grants from the NIH (R01DK093587, R01DK101379, and R00DK085330 to Y. Xu; P01 DK088761 to D.J. Clegg; T32CA059268 to S.A. Khan; NIHDK59820 to S.Y. Tsai; DK5U19DK062434 to F.J. DeMayo; R01DK092605 to Q. Tong), Marcadia Biotech (B. Yang and R. DeMarchi), American Diabetes Association (1-11-BS-180 to Y. Xu and \#7-13-JF-61 to Q. Wu), and American Heart Association awards to P. Xu and to Q. Tong. Measurements of body composition, food intake and energy expenditure were performed in the Mouse Metabolic Research Unit (MMRU) at the USDA/ARS Children's Nutrition Research Center, which is supported by funds from the USDA ARS (www. bcm.edu/cnrc/mmru). The authors acknowledge the assistance of Firoz Vohra and the MMRU Core Director, Marta Fiorotto. The behavioral tests were performed at the Mouse Neurobehavior Core, Baylor College of Medicine, which is supported by NIH P3OHD024064. We also thank Bryan L. Roth (University of North Carolina) and Martin G. Myers Jr. (University of Michigan) for providing viral strains.

Address correspondence to: Yong $\mathrm{Xu}, 1100$ Bates Street, $\mathrm{Rm}$ 8070, Houston, Texas 77030, USA. Phone: 713.798.7199; E-mail: yongx@bcm.edu.
1. Riant E, Waget A, Cogo H, Arnal JF, Burcelin R, Gourdy P. Estrogens protect against high-fat diet-induced insulin resistance and glucose intolerance in mice. Endocrinology. 2009;150(5):2109-2117.

2. Wallen WJ, Belanger MP, Wittnich C. Sex hormones and the selective estrogen receptor modulator tamoxifen modulate weekly body weights and food intakes in adolescent and adult rats. J Nutr. 2001;131(9):2351-2357.

3. Gao Q, et al. Anorectic estrogen mimics leptin's effect on the rewiring of melanocortin cells and Stat 3 signaling in obese animals. Nat Med. 2007;13(1):89-94.

4. Heine PA, Taylor JA, Iwamoto GA, Lubahn $\mathrm{DB}$, Cooke PS. Increased adipose tissue in male and female estrogen receptor-alpha knockout mice. Proc Natl Acad Sci U S A. 2000;97(23):12729-12734.

5. Bryzgalova G, et al. Evidence that oestrogen receptor-alpha plays an important role in the regulation of glucose homeostasis in mice: insulin sensitivity in the liver. Diabetologia. 2006;49(3):588-597.

6. Ogawa S, Chan J, Gustafsson JA, Korach KS,
Pfaff DW. Estrogen increases locomotor activity in mice through estrogen receptor alpha: specificity for the type of activity. Endocrinology. 2003;144(1):230-239.

7. Xu Y, et al. Distinct hypothalamic neurons mediate estrogenic effects on energy homeostasis and reproduction. Cell Metab. 2011;14(4):453-465.

8. Musatov S, et al. Silencing of estrogen receptor alpha in the ventromedial nucleus of hypothalamus leads to metabolic syndrome. Proc Natl Acad Sci US A. 2007;104(7):2501-2506.

9. Martinez de Morentin PB, et al. Estradiol regulates brown adipose tissue thermogenesis via hypothalamic AMPK. Cell Metab. 2014;20(1):41-53.

10. Asarian L, Geary N. Estradiol enhances cholecystokinin-dependent lipid-induced satiation and activates estrogen receptoralpha-expressing cells in the nucleus tractus solitarius of ovariectomized rats. Endocrinology. 2007;148(12):5656-5666.

11. Santollo J, Torregrossa AM, Eckel LA. Estradiol acts in the medial preoptic area, arcuate nucleus, and dorsal raphe nucleus to reduce food intake in ovariectomized rats. Horm Behav.
2011;60(1):86-93.

12. Cao X, et al. Estrogens stimulate serotonin neurons to inhibit binge-like eating in mice. J Clin Invest. 2014;124(10):4351-4362.

13. Merchenthaler I, Lane MV, Numan S, Dellovade TL. Distribution of estrogen receptor alpha and beta in the mouse central nervous system: in vivo autoradiographic and immunocytochemical analyses. J Comp Neurol. 2004;473(2):270-291.

14. Donohoe TP, Stevens R. Modulation of food intake by amygdaloid estradiol benzoate implants in female rats. Physiol Behav. 1981;27(1):105-114.

15. Alexander GM, et al. Remote control of neuronal activity in transgenic mice expressing evolved G protein-coupled receptors. Neuron. 2009;63(1):27-39.

16. Ferguson SM, et al. Transient neuronal inhibition reveals opposing roles of indirect and direct pathways in sensitization. Nat Neurosci. 2011;14(1):22-24.

17. Kublaoui BM, Holder JL, Holder JL Jr, Gemelli T, Zinn AR. Sim1 haploinsufficiency impairs melanocortin-mediated anorexia and activation of paraventricular nucleus neurons. Mol Endocrinol. 
2006;20(10):2483-2492.

18. Michaud JL, et al. Sim1 haploinsufficiency causes hyperphagia, obesity and reduction of the paraventricular nucleus of the hypothalamus. Hum Mol Genet. 2001;10(14):1465-1473.

19. Xi D, Gandhi N, Lai M, Kublaoui BM. Ablation of Sim1 neurons causes obesity through hyperphagia and reduced energy expenditure. PLoS One. 2012;7(4):e36453.

20. Balthasar N, et al. Divergence of melanocortin pathways in the control of food intake and energy expenditure. Cell. 2005;123(3):493-505.

21. Feng Y, Manka D, Wagner KU, Khan SA. Estrogen receptor-alpha expression in the mammary epithelium is required for ductal and alveolar morphogenesis in mice. Proc Natl Acad Sci U S A. 2007;104(37):14718-14723.

22. Castaneda TR, et al. Obesity and the neuroendocrine control of energy homeostasis: the role of spontaneous locomotor activity. Journal Nutr. 2005;135(5):1314-1319.

23. Kaiyala KJ, Morton GJ, Leroux BG, Ogimoto K, Wisse B, Schwartz MW. Identification of body fat mass as a major determinant of metabolic rate in mice. Diabetes. 2010;59(7):1657-1666.

24. Finan B, et al. Targeted estrogen delivery reverses the metabolic syndrome. Nat Med. 2012;18(12):1847-1856.

25. Gu G, Roland B, Tomaselli K, Dolman CS, Lowe C, Heilig JS. Glucagon-like peptide-1 in the rat brain: distribution of expression and functional implication. JComp Neurol. 2013;521(10):2235-2261.

26. Stauffer SR, et al. Pyrazole ligands: structure-affinity/activity relationships and estrogen receptor-alpha-selective agonists. JMed Chem. 2000;43(26):4934-4947.

27. Sohn JW, Xu Y, Jones JE, Wickman K, Williams KW, Elmquist JK. Serotonin $2 \mathrm{C}$ receptor activates a distinct population of arcuate pro-opiomela- nocortin neurons via TRPC channels. Neuron. 2011;71(3):488-497.

28. Leinninger GM, et al. Leptin action via neurotensin neurons controls orexin, the mesolimbic dopamine system and energy balance. Cell Metab. 2011;14(3):313-323.

29. Callewaert F, et al. Differential regulation of bone and body composition in male mice with combined inactivation of androgen and estrogen receptor-alpha. FASEB J. 2009;23(1):232-240.

30. Grumbach MM, Auchus RJ. Estrogen: consequences and implications of human mutations in synthesis and action. JClin Endocrinol Metab. 1999;84(12):4677-4694.

31. Smith EP, et al. Estrogen resistance caused by a mutation in the estrogen-receptor gene in a man. N Engl J Med. 1994;331(16):1056-1061.

32. Jones ME, et al. Aromatase-deficient (ArKO) mice have a phenotype of increased adiposity. Proc Natl Acad Sci U S A . 2000;97(23):12735-12740.

33. Wu MV, et al. Estrogen masculinizes neural pathways and sex-specific behaviors. Cell. 2009;139(1):61-72.

34. Wang G-J, et al. Decreased brain aromatase availability in overweight humans. J Nucl Med. 2011;52(suppl 1):340.

35. Malyala A, Zhang C, Bryant DN, Kelly MJ, Ronnekleiv OK. PI3K signaling effects in hypothalamic neurons mediated by estrogen. J Comp Neurol. 2008;506(6):895-911.

36. Park CJ, et al. Genetic rescue of nonclassical $\mathrm{ER} \alpha$ signaling normalizes energy balance in obese Era-null mutant mice. J Clin Invest. 2011;121(2):604-612.

37. Hong W, Kim DW, Anderson DJ. Antagonistic control of social versus repetitive self-grooming behaviors by separable amygdala neuronal subsets. Cell. 2014;158(6):1348-1361.

38. Wu SP, Lee DK, Demayo FJ, Tsai SY, Tsai MJ.
Generation of ES cells for conditional expression of nuclear receptors and coregulators in vivo. $\mathrm{Mol}$ Endocrinol. 2010;24(6):1297-1304.

39. Cheung AF, Dupage MJ, Dong HK, Chen J, Jacks T. Regulated expression of a tumor-associated antigen reveals multiple levels of T-cell tolerance in a mouse model of lung cancer. Cancer Res. 2008;68(22):9459-9468.

40. Xu Y, et al. PI3K signaling in the ventromedial hypothalamic nucleus is required for normal energy homeostasis. Cell Metab. 2010;12(1):88-95.

41. Butler AA, Kozak LP. A recurring problem with the analysis of energy expenditure in genetic models expressing lean and obese phenotypes. Diabetes. 2010;59(2):323-329.

42. Enriori PJ, Sinnayah P, Simonds SE, Garcia Rudaz C, Cowley MA. Leptin action in the dorsomedial hypothalamus increases sympathetic tone to brown adipose tissue in spite of systemic leptin resistance. J Neurosci. 2011;31(34):12189-12197.

43. Hill JW, et al. Acute effects of leptin require PI3K signaling in hypothalamic proopiomelanocortin neurons in mice. J Clin Invest. 2008;118(5):1796-1805.

44. Wang G, et al. Evidence that estrogen directly and indirectly modulates $\mathrm{C} 1$ adrenergic bulbospinal neurons in the rostral ventrolateral medulla. Brain Res. 2006;1094(1):163-178.

45. Ren H, et al. FoxO1 target Gpr17 activates AgRP neurons to regulate food intake. Cell. 2012;149(6):1314-1326.

46. Pinto S, et al. Rapid rewiring of arcuate nucleus feeding circuits by leptin. Science. 2004;304(5667):110-115.

47. Merlo S, Frasca G, Canonico PL, Sortino MA. Differential involvement of estrogen receptor alpha and estrogen receptor beta in the healing promoting effect of estrogen in human keratinocytes. JEndocrinol. 2009;200(2):189-197. 